Historic, archived document

Do not assume content reflects current scientific knowledge, policies, or practices. 


$$
+
$$





\section{For Dealers and Nurserymen Only}

To customers of good credit invoices for seeds, plants and bulbs, with exceptions hereafter noted, will be subject to $11 / 2 \%$ discount if paid within 10 days of date of invoice, or net 60 days. Grass, clover and farm seeds, onion sets, potatoes, fertilizers, insecticides and sundries are invariably net. All shipments f. o. b. Rochester. Satisfactory references required from new or unknown correspondents. Ledger accounts cannot be opened for first orders of less than \$5.00; such orders should be accompanied by cash.

Perishable goods will be sent C. O. D. only when a partia l remittance is received in advance as a guarantee of acceptance of shipment, and all C. O. D. charges are to be paid by purchaser. Prices herein are subject to change without notice. A nominal charge extra for bags and boxes. Retail packets, except novelties, are subject to $40 \%$ discount where less than 100 packets are ordered. In quantities of 100 or more, retail packets are subject to $50 \%$ discount.

Ounces and fractions thereof of vegetable seed $40 \%$ discount from retail prices. Flower seeds by the ounce and pound and Vegetable seeds by the pound and bushel are priced net in the body of this catalogue, $-1 / 4$ pound at the pound rate; $1 / 4$ bushel at the bushel rate. Smaller quantities at a slight advance. No quantity of seed packed from bulk for less than ten cents. When smaller quantities are required use the five or ten cent papers, taking advantage of the discounts indicated above.

Purchasers will please send explicit shipping instructions with each order. Where no such instructions are given we shall exercise our best judgment in the matter; but when goods have been so delivered at the freight or express station, in Rochester, in first-class condition, addressed as ordered, we disclaim all responsibility for any accident, damage, or delay en route. When goods are desired sent by mail, postage must be added at the rate of $10 \mathrm{c}$ per pound, otherwise we will deduct enough from the order to cover postage or add the postage to invoice as the case may require.

While we exercise great care to send out Seeds, Plants and Bulbs of the best types and of high germination, we give no warranty, express or implied, as to description, quality, productiveness, or any other matter, of any seeds, bulbs or plants we send out, and we will not be in any way responsible for the crop. If the purchaser does not accept goods on these terms they are at once to be returned and any payments made in advance for same will be refunded.

\section{JAMES VICK'S SONS}




\section{VICK'S TRADE LIST}

\section{Vegetable Seeds}

\section{Per 10 Lbs. and over \\ Lb. Per Lb.}

Green Globe. . . . . . . . . . \$3.50

\section{ASPARAGUS}

Columbian Mammoth....... . .60

Conover's Colossal. .......... . .60

Palmetto................ .60

Vick's New Mammoth........ . .60

Washington "Rustless". . . . . . 1.35

\section{$\$ 3.25$}

\section{ASPARAGUS ROOTS}

Conover's Roots, two year No. 1

$\$ 1.25$ per $100 ; \$ 10.00$ per 1,000

Palmetto Roots, two year No. 1 $\$ 1.25$ per $100 ; \$ 10.00$ per 1,000

Vick's New Mammoth, Roots two year No. 1 . $\$ 1.25$ per 100 ; $\$ 10.00$ per 1,000

Washington "Rustless" Roots, $\$ 2.00$ per $100 ; \$ 18.00$ per 1,000 .

\section{BEANS, DWARF GREEN-POD}

$\begin{array}{ccc}\text { Per } & 20 & \text { Per } \\ \text { Lb. } & \text { Lbs. } & \text { Bu. } \\ & \text { or } & 60 \text { Libs. } \\ & \text { more } & \\ \text { Per } & \\ \text { Lb. } & \end{array}$

Bountiful.................\$.19

Dwarf Horticultural. . . . . . . . . . .17

Extra Early Refugee.......... . .17

Extra Early Red Valentine.... . .18

Full Measure................ . 19

"Giant Stringless"; Green-pod.. . $\quad .19$

Keeney's Stringless Refugee. ... . .21

Long Yellow Six Weeks........ . . 18

Refugee, 1,000 to $1 \ldots \ldots \ldots \ldots . .18$

Stringless Green-pod (Burpee's). $\quad .19$

$\$ .18 \$ 10.80$

$\begin{array}{ll}.16 & 9.60\end{array}$

$.16 \quad 9.60$
.17

$\begin{array}{ll}.17 & 10.20\end{array}$

$.18 \quad 10.80$

$.18 \quad 10.80$

$.20 \quad 12.00$

$.17 \quad 9.60$
.17

$.17 \quad 10.20$

$.18 \quad 10.80$

\section{BEANS, DWARF WAX-POD}

Brittle Wax.............. . .21

Burpee's Kidney Wax........ . .21

Davis White Wax........... .21

Hardy Wax................ .21

Improved Rustless Golden Wax. $\quad .20$

Keeney's Rustless Golden Wax. $\quad .20$

Pencil Pod Black Wax......... .20

Prolific Improved Black Wax... .20

Refugee Wax.............. . 19

Sure Crop Stringless Wax. . . . . . . 21

Wardwell's Kidney Wax...... . .21

\section{BEANS, FIELD}

Pea or Navy . . . . . . . . . . . .12

Red Kidney.................. .17

White Kidney.............. . 19

Marvelous................ .19 
BEANS, DWARF OR BUSH

20 lbs. or Per bu LIMA

Per Lb. more Per !b. 60 lbs.

Henderson's Bush Lima . . . . . .\$ .20

Improved Bush Lima (Burpee's) .24

Fordhook Bush Lima......... . .27

$\$ .19$

$\$ 11.40$

.23

13.80

Prolific Bush Lima (Wood's). . . .21

.26

15.60

$.20 \quad 12.00$

\section{BEANS, POLE OR RUNNING}

Early Golden Cluster Wax.... . .21

Old Homestead (Kentucky Wonder $) \ldots \ldots \ldots \ldots \ldots \ldots \ldots .18$

Scarlet Runner............ . .21

Lima-Extra Early Jersey . . . . . .21

Lima-King of the Garden.... . .21

Giant Podded Pole Lima...... . .25

Crimson Globe........... \$ .45

Crosby's Early Egyptian...... . .55

Detroit Dark Red, Best Main Crop.................. .55

Early Wonder, Splendid Extra Early.................. .60

Eclipse, Extra Early . . . . . . . . .50

Improved Early Blood Turnip. . $\quad .50$

Long Smooth Blood Red....... . .40

Rochester................. .50

Vick's Early Market........ . .50

\section{SUGAR BEETS}

Klein Wanzleben....................

White French. . . . . . . . . . . . 35

MANGEL WURZEL

Danish Taaroje, extra fine for heavy soil................

Danish Sludstrup, extra fine for deep soil. ...............

Giant Half Sugar Rose. . . . . . .

Golden Giant, great cropper... .

Golden Tankard, splendid for dairy cattle..............

Giant Yellow, intermediate.....

Vick's Mammoth Long Red....

\section{SWISS CHARD (Salad Beet)}

Large-ribbed Silver.......... . .50

Large-ribbed Yellow ........... . . .45

Lucullus, an improved variety large stalks. . . . . . . . . . . . .

BORECOLE (See Kale page 6)

\section{BROCCOLI}

White Cape..... Per Oz.\$ .50 5.00

\section{BRUSSELS SPROUTS}

Improved Dwarf or Dalkeith... 
Per

$\mathrm{Lb}$.

\section{CHICORY}

Large-rooted Long Magdeburg. . $\$ 1.00$

Witloof French Endive, a fine

salad ............... 1.35
10 Lbs. and over per Lb.

$\$ .90$

1.25

CORN SALAD 1.00

\section{CABBAGE}

Allhead Early............. 1.35

All Seasons (choice strain).... 1.35

Autumn King or World Beater . 1.35

Charleston Wakefield (extra

large $) \ldots \ldots \ldots \ldots \ldots \ldots \ldots \ldots$

Chinese (Pe Tsai) . . . . . . 1.50

Copenhagen Market. American grown .............. 1.60

Copenhagen Market. Best Danish grown............. 2.00

Dwarf Amager or Vick's Short

Stemmed Danish Ballhead... 1.35

Danish Ballhead, best long keep-

ing strain .............. 1.35

Danish Stonehead or Round Red 1.60

Extra Early Drumhead. . . . . . 1.50

Early Jersey Wakefield....... 1.75

Early Spring . . . . . . . . . 1.50

Early Winnigstadt......... 1.50

Glory of Enkhuizen......... 1.75

Improved American Savoy..... 1.50

Improved Early Summer. . . . . 1.50

Large Late Drumhead American 1.35

Louisville Drumhead. ........ 1.50

Mammoth Rock Red or Stone. . 1.60

Premium Late Flat Dutch . . . . 1.50

Surehead................ 1.35

Succession, Improved Strain.... 1.35

\section{CARROT}

Chantenay Stump-rooted ..... . .75

Vick's Coreless. ............ 1.10

Danvers Half Long Orange..... . .75

Earliest French Forcing........ .75

Early Scarlet Short Horn..... . . .75

Oxheart or Guerande......... . .75

Half Long Scarlet Nantes. . . . . . . $\quad .75$

Improved Long Orange........ . . .75

St. Valerys Intermediate, fine long red.............. .75

White Belgian Green-top . . . . . . . .45

Orange Belgian.............. .55

Butter................. 1.10 
CAULIFLOWER, Continued.

Per Oz. Per Lb.

Erfurt Earliest Dwarf . . . . . . \$1.25

Early Snowball............ 1.25

Extra Early Paris, for forcing.. . $\quad .75$

$\$ 15.00$

15.00

7.50

Vick's Danish Giant or Dry

Weather............... 1.50

Vick's Ideal. . . . . . . . . . . . . 1.25

Veitch's Autumn Giant....... . .50

Vick's Superior. . . . . . . . . . 1.25

18.00

15.00

5.00

15.00

Per

CRESS or PEPPER GRASS

$10 \mathrm{Lbs}$. and
over per $\mathrm{Lb}$.

Fine Curled True Pepper Grass. \$ .35

Broad Leaved Garden......... . . 35

True Water............. 2.75

\section{CELERY}

Golden Dwarf Self-blanching

(French Grown) Old Dwarf

Strain............... 6.00

5.75

Golden Dwarf Self-blanching

(American Grown)......... 2.75

Celeriac, Large Smooth Prague. . 1.50

Columbia Rich Golden Yellow. . 1.50

Easy Blanching, Truckers va-

riety. ............. 2.75

Giant Golden Heart. . . . . . . . . 1.10

Giant Pascal.............. 1.10

Iunroved White Plume....... 1.75

Winter Queen............. 1.50

Celery for flavoring . . . . . . . . . 50

Golden Plume or Wonderful... . 12.00

2.50

1.40

1.40

2.50

1.00

1.00

1.60

1.40

.45

11.00

\section{CORN-SWEET OR_SUGAR}

20 Lbs. Per

or more $\mathrm{Bu}$.

Per Lb. Per Lb. $50 \mathrm{Lbs}$

Black Mexican . . . . . . . . . . \$.14

Bantam Evergreen. . . . . . . . . . . 14

Country Gentleman. ......... . .16

Dreers Aristocrat. . . . . . . . . . . . . . . . . .

Early Dawn............... . . .14

Early Golden Bantam......... . . 14

Early Minnesota........... . . . . . . . .

Howling Mob............ . 14

Vick's Golden Nugget, extra fine. $\quad .14$

Early Evergreen........... . . 16

Extra Early Red Cory.... . . . . . . 14

Mammoth White Cory....... . . 14

Vick's Extra Early Morning Dew .14

Pocahontas, very early . . . . . . . .14

Stowell's Evergreen .......... $\quad .16$

Vick's Earliest of All . . . . . . . . . . . . . . .

Whipple's Early White....... . . .

Whipple's Early Yellow....... . . 18

$.11 \$ 6.00$

$.12 \quad 6.00$

$.14 \quad 7.00$

$.12 \quad 6.00$

.126 .00

.126 .00

$.12 \quad 6.00$

$.12 \quad 6.00$

$.12 \quad 6.00$

$.14 \quad 7.00$

$.12 \quad 6.00$

$.12 \quad 6.00$

$.12 \quad 6.00$

$.12 \quad 6.00$

$.14 \quad 7.00$

$.12 \quad 6.00$

$.17 \quad 8.50$

$.17 \quad 8.50$ 
Per Bu. $10 \mathrm{Bu}$. or over of Per Bu.

56 Lbs.

CORN, FIELD

Genesee Valley Early Yellow, 8 rowed flint............\$2.40

Improved Leaming, Early Yellow Dent. .............. 2.25

Longfellow, Early Yellow 8rowed Flint........... 2.40 Vick's Snow Capped Gold Mine. 1.85

Early Northern White Dent... . 2.40

\section{POP CORN (Shelled)}

Lb.

Queen's Golden..........\$ .14

Red Beauty...........................

White Rice............... . 14

Japanese Hulless. . . . . . . . . . . . .15

$\$ 2.30$

2.15

2.30

1.75

2.30

10 Lbs. and over Per Lb.

\section{CUCUMBER}

Per

Lb.

Abundance Forcing . . . . . . . \$4.50

Chicago Pickling........... . .85

Cool and Crisp............ .90

Davis Perfect. . . . . . . . 1.25

Early Fortune............. . 1.10

Early Green Cluster.......... . .85

Earliest of All ........... .9. 90

Extra Early Long Green. . . . . . 1.35

Green Prolific or Boston Pickling................... 85

Improved Early White Spine

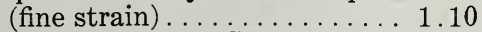

Improved Long Green (extra choice) . ............... 1.35

Japanese Climbing. . . . . . . . 1.10

Kirby's Stays Green........... 1.10

Thorburn's Everbearing . . . . . . . . .85

Vick's Perfection White Spine. . 1.10

Vick's Lemon............... 1.25

Arlington White Spine........ 1.10

$$
\begin{array}{r}
\$ .12 \\
.12 \\
.12 \\
.14
\end{array}
$$

10 Lbs. and over Per Lb.

DILL. .45

\section{EGG PLANT}

Black Beauty. . . . . . . . . . . 3.25

Improved New York Purple

Spineless............... 3.25

\section{ENDIVE}

Broad-Leaved Batavian....... . .65

Green Curled................ .65

Moss Curled . ................. .70

White Curled............... .65

French (Witloff Chicory)...... .95

$\$ .80$

.85

1.15

1.00

.80

.85

1.25

.80

1.00

1.25

1.00

1.00

.80

1.00

1.15

1.00

.40

3.00

3.00

\section{FENNEL}

Florence.............. 1.00

Giant Messina (Best for market garden) $\ldots \ldots \ldots \ldots \ldots \ldots 1.00$ 


\section{KALE or BORECOLE}

Per

Lb.

Dwarf Green Curled or Superior Scotch Kale.............\$.60

Half Dwarf Moss Curled....... .65

Siberian............. .55

Tall Green Curled or Scotch Kale

KOHL-RABI

Early White Vienna.......... 1.10

Early Purple Vienna......... 1.10

\section{LEEK}

Broad Flag, American........ 1.10

Monstrous Carenton . . . . . . . . 1.10

LETTUCE

All Seasons.............. .75

Big Boston, Leading Commercial Variety................ .80

Big Boston, Vick's Special Strain 1.00

Black-Seeded Simpson......... .85

Crisp as Ice... . . . . . . . . . . 1.00

Early Curled Simpson or Silesia. .85

Golden Queen Forcing......... 1.00

Grand Rapids Forcing, Improved 1.00

Iceberg. . . . . . . . . . . . . 1.25

Improved Early Hanson. . . . . . . .85

May King. . . . . . . . . . . 1.00

New York or Los Angeles...... 1.75

Paris White Cos, Self-folding... 1.00

Prize Head.............. 1.00

MELON, MUSK

Bender's Surprise . . . . . . . . 1.25

Burrell's Gem, pink flesh, Rocky

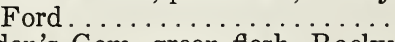

Eden's Gem, green flesh, Rocky Ford ..................

Early Knight, Best early...... . . .75

Extra Early Hackensack...... . . .75

Emerald Gem, orange flesh..... $\quad .75$

Honey Dew, green flesh....... $\quad .90$

Jenny Lind, extra early....... . .75

Montreal Market (Montreal Nutmeg)................ .75

Osage or Miller's Cream...... $\quad .75$

Paul Rose or Petosky........ $\quad .70$

Rocky Ford, green flesh (Netted Gem) ................. .75

Rocky Ford, gold lined ....... $\quad .75$

Irondequoit............... 1.50

Vick's Prolific Nutmeg.......... $\quad .75$

MELON, WATER

Citron, for preserves, red seed. . .55

Cole's Early... . . . . . . . . . . . .55

Cuban Queen............. . .50

Florida Favorite. . . . . . . . . . . 50

Fordhook Early............. . . .55

Georgia Rattlesnake......... . .50

Ice Cream or Peerless. . . . . . . . . . .55

Kleckley Sweets. . . . . . . . . .55

Prize Jumbo . . . . . . . . . . . . . .50

Sweetheart.............. .50

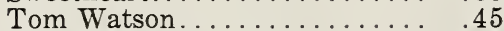

Vick's Extra Early. . . . . . . . . . .55
$10 \mathrm{Lbs}$. and over Per Lb.

$\$ .55$

.50

.60

1.00

1.00

1.00

1.00

.70

.75

.90

.80

.90

.80

.90

.90

1.15

.80

.90

1.65

.90

.90

1.15

.70

.70

.70

.70

.70

.80

.70

.70

.70

.65

.70

.70

1.40

.70 
MUSHROOM SPAWN

Bottle Spawn is the latest scien-

Per

Per Case

Bottle

12 Bottles tific development of Spawn culture. By actual experience under growing conditions it has proven the most prolific Spawn ever developed. Each bottle will plant about fifty square feet. This Spawn must be used while fresh. It is shipped by us direct from the laboratories on receipt of your order. Will keep for several months at a temperature of 32 degrees. Full cultural directions with each shipment. Shipped express, not prepaid..........\$1.00

Pure Culture Brick Spawn.

The best and most reliable brick Spawn in cultivation. 25 or more

\section{MUSTARD}

Chinese Broad Leaf .......... \$ .35

Southern Giant Curled.......... .35

Ostrich Plume............. . 35

\section{OKRA}

Perkins' Mammoth Green Pod. . . .35

White Velvet............. . .35
10 Lbs. and over Per Lb.

\section{ONION}

Australian Brown........... \$3.00

Crystal White Wax........... 5.00

Danvers Yellow Globe, extra selected.

Danvers Yellow Globe, commercial stock. .............

Extra Early Red Flat.

Large Red Wethersfield........

Large Yellow Dutch or Strasburg Mammoth Prize Taker........

Red Bermuda..............

Silver King or White Garganus.

Southport Red Globe..........

Southport Yellow Globe, selected

Southport White Globe........

White Bermuda..............

White Portugal or Silver Skin. .

White Queen..............

Ebenezer or Japanese.........

\section{ONION SETS}

$$
\begin{array}{r}
\$ .32 \\
.32 \\
.32
\end{array}
$$

Our Onion Sets are carefully screened, clean, sound and dry.

Yellow....................

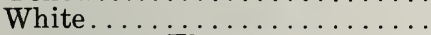

Japanese or Ebenezer. 

over Per Lb.

\section{PARSNIP}

Elcombes Giant...........\$ .45

Improved Guernsey.......... .45

Long Hollow Crown........ .45

\section{PARSLEY}

Champion Moss Curled....... .45

Fine Triple Curled.......... . .45

Fern-leaved ............. .45

Hamburg Large-rooted . . . . . . . .45

Vick's Exquisite Moss Curled... . .45

Plain Leaved. . . . . . . . . . . . . .45

\section{PEAS}

The average weight of Peas per bushel is $56 \mathrm{lbs}$. for the wrinkled varieties and $60 \mathrm{lbs}$. for smooth sorts. In the list below we have marked the wrinkled varieties with a star $(*)$. 20 Lbs. and over

$\begin{array}{llll} & \text { Per } & \text { Per } & \text { Per } \\ \text { Early Varieties } & \text { Lb. } & \text { Lb. } & \text { Bu. }\end{array}$

Alaska. One of the most popular extra early varieties with canners and market gardeners. Half dwarf . ...........\$.15

*American Wonder. Excellent second early. Very dwarf....

*Carter's Early Eight Weeks. One of the best new varieties. The earliest wrinkled Pea of good quality. Very dwarf....

*Early Morn. Slightly earlier than Gradus. Superior quality. Dwarf....................

*Extra Early Premium Gem
Improved Little Gem. Dwarf

* Gradus (Prosperity). One of the most popular early sugar Peas. Half dwarf. . . . . . . . . . . . .

First and Best. Very early.

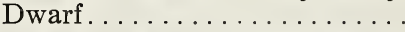

*Nott's Excelsior. Early, dwarf. Superior quality............

*Surprise. Excellent market variety. Half dwarf . . . . . . . . . .

*Laxtonian. Very äwarf. Large pods. Early.............

*Little Marvel. Early. Very

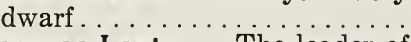

$\begin{array}{lll}.16 & .15 & 8.40\end{array}$

$\begin{array}{lll}18 & .17 \quad 9.52\end{array}$

$\begin{array}{lll}.18 & .17 \quad 9.52\end{array}$

$\begin{array}{lll}.15 & .14 & 7.84\end{array}$

$.17 \quad .16 \quad 8.96$

$\begin{array}{lll}.16 & .15 \quad 9.00\end{array}$

$.16 \quad .15 \quad 8.40$

$\begin{array}{lll}.16 & .15 \quad 8.40\end{array}$

$.21 \quad .20 \quad 11.20$

$.19 \quad .18 \quad 10.08$

*Thomas Laxton. The leader of the extra earlies. Very dwarf. Large pods................

Vick's First Choice. One of the earliest half dwarf...........

Vick's Extra Early. One of the earliest half dwarf...........

*World's Record. Earlier than Gradus. Half dwarf........ 
PEAS, Early Varieties-Con.

\section{Later Varieties}

*Alderman (Admiral Dewey) Dark podded Telephone type.

Tall. Late.............. \$

*Abundance. Second early or mid-season. Dwarf. .........

*Bliss Everbearing. A standard main crop variety. Vines about three feet...............

*Boston Unrivalled. Larger pods and superior to old Telephone. Tall. Late. . . . . . . . . . . . .

Dwarf Gray Sugar. Edible pod

* Champion of England. One of the best flavored late Peas. Tall.....................

*Improved Stratagem. An old time favorite. Dwarf. Large pods. Late. ................

Canada Field................

Large Black-eyed Marrowfat. Tall. Late...............

Mammoth Melting Sugar. Edible pod. Tall. Late.....

*Telephone. Standard late market variety. Tall............

*Vick's Daisy. Dwarf telephone type. Late...............

\section{PEPPER}

Per or over Per

Lb. Per Lb. Bu.
$\$ .16 \$ 8.96$

$\begin{array}{lll}.16 & .15 & 8.40\end{array}$

$\begin{array}{lll}.16 & .15 & 8.40\end{array}$

$\begin{array}{lll}.17 & .16 & 8.96\end{array}$

$\begin{array}{lll}.16 & .15 \quad 9.00\end{array}$

$\begin{array}{lll}.17 & .16 \quad 8.96\end{array}$

$\begin{array}{lll}.17 & .16 & 8.96\end{array}$

$\begin{array}{lll}.08 & .07 & 4.20\end{array}$

$\begin{array}{lll}.11 & .10 \quad 6.00\end{array}$

$\begin{array}{lll}.22 & .21 & 12.60\end{array}$

$.17 \quad .16 \quad 8.96$

$\begin{array}{lll}.18 & .17 \quad 9.52\end{array}$

10 Lbs. and over Per Lb.

Celestial, bright scarlet. . . . . $\$ 2.50$

Chinese Giant. . . . . . . . . . . . 4.25

Chili, very small, for pepper sauce...............2.25

Golden Dawn or Queen, bright yellow ................ 3.50

Large Bell or Bull Nose...... . 2.50

Long Red Cayenne............ 2.75

Neopolitan extra early sweet. . 2. 2.50

Pimento extra fine sweet...... 2.25

Royal King.................2.00

Red Cluster. ............ 2.25

Ruby Giant, large sweet, fine 2.50

Ruby King, bright red......... 2.50

Sweet Mountain or Mammoth.. 2.50

Vick's Giant Red (Ruby Giant). 2.50

Harris Earliest. . . . . . . . . 3.00

\section{PUMPKIN}

Connecticut Field.......... . .45

Golden Oblong. ............. . .70

King of the Mammoths or Jumbo .85

Large Tours or Mammoth..... $\quad .85$

Large Sweet Cheese or Kentucky

Field ................. .50

Tennessee Sweet Potato. . . . . . . .75

Small Sugar or Pie........... $\quad .60$

Winter Luxury. . . . . . . . . . .60 


\section{RADISH}

Vick's Early Scarlet Globe, best forcing variety ever introduced . . . . . . . . . . . . . . $\$ .50$

All Seasons............... .55

Cooper's Sparkler........... . .45

Crimson Giant............. .45

French Breakfast........... .45

Icicle................... .45

Improved Long Scarlet Short Top...................

$\quad$ Long Brightest Scarlet $\quad .45$

Long White Vienna or Lady Finger..................

Scarlet Turnip, White tip, for forcing..................

Vick's "Three Weeks"...........

White Strasburg . . . . . . . . 50

RADISH Winter Varieties

Black Spanish Winter, round... .45

Black Spanish Winter, long.... . .45

California Mammoth White

Winter................. .45

Chinese Rose Winter............. $\quad .45$

Japanese Sahurajima Mammoth .60

Celestial or Chinese White.... . .45

Chinese Round Scarlet....... .55

\section{RHUBARB}

Linnaeus................. $\quad .85$

Victoria.................... .85

RHUBARB ROOTS

Doz.

Victoria or Linnaeus. . . . . . . . \$ .75

Strawberry, true............ 1.00

\section{SALSIFY}

Lb.

Mammoth Sandwich Island.... \$1.50

\section{SPINACH}

Bloomsdale, Savoy-leaved (Nor-

folk) ............... \$ .20

Improved Thick-leaved........ $\quad .20$

King of Denmark........... . .22

Monstrous or Viroflay........... $\quad .20$

New Zealand .............. .45

Prickly or Fall. .................

Round or Summer Broad-Leaf. . $\quad .20$

Triumph............... .20

Victoria, fine long standing sort. $\quad .20$

Vick's All Seasons............. .20

SQUASH

Boston Marrow...........\$ .55

Chicago Warted Hubbard...... $\quad .80$

Delicious .................. $\quad .80$

Early White Bush Scallop (Patty Pan) ................. . 80

Fordhook............... $\quad .80$

Improved Hubbard. . . . . . . . . $\quad .75$

10 Lbs. and over Per Lb.

10 Lbs. and over per Lb.

$\$ 1.40$ 
SQUASH-Continued.

Mammoth Yellow Summer Crookneck.............

Mammoth White Bush (Silver Custard) ................ .80

New Golden Hubbard. . . . . . . $\quad .80$

Mammoth Chili. ............ .85

Mammoth Yellow Bush Scallop. $\quad .60$

Cocozelle di Nopoli. . . . . . . . . $\quad .85$ TOMATO

Acme (Improved Strain) . . . . . 2.00

Beauty ..................2.50

Bonny Best................. 2.25

Chalk's Early Jewel (Vick's Se-

lected) ............... 2.25

Dwarf Champion. . . . . . . . . 3.00

Dwarf Stone............ 3.00

Earliana-Special Strain...... 2.75

Earliana, Commercial Stock.... 2.25

Favorite................. 2.00

Greater Baltimore. . . . . . . . . . 2.10

Improved Trophy ........... 1.50

John Baer. . . . . . . . . . . . . 2.10

Keyes' Prolific............... . 2.00

Large Yellow or Golden Queen. . 3.00

Magnus . . . . . . . . . . . . . . 2.00

Perfection............... 2.00

Ponderosa ............... 4.00

Royal Purple............... 1.50

Royal Red................. 1.50

Stone, Improved. . . . . . . . . 1.75

Sterling Castle............. 3.00

TOMATO Small Fruited Varieties.

Husk or Strawberry or Winter Cherry ............... 3.75

Peach, Yellow.............. 3.50

Peach, Red.............. . 3.50

Pear-shaped Red............ 4.00

Pear-shaped Yellow......... 4.00

Plum-shaped Yellow......... . 4.00

Red Cherry............... 4.00

Red Currant............... 4.75

Yellow Cherry............ 4.00 TOBACCO

Per

$\mathrm{Oz}$.

Connecticut Seed-Leaf........\$\$.25

Havana, Cuban grown (Vuslto de Abajo).............. .25

White Burley............... .25

Per

Lb.

TURNIP. Table Varieties.

Early White Flat Dutch...... \$ .35

Early White Six Weeks, or Snowball ................. .40

Extra Early Purple Top Milan. . $\quad .50$

Extra Early White Milan..... . .50

Golden Ball or Orange Jelly.... . . .35

Pomeranian White Globe. .... . .30

Purple Top, Strap-leaved..... . . 35
10 Lbs. and over Per Lb.

$\$ .75$

.75

.75

.80

.55

.80

1.90

2.40

2.15

2.15

2.90

2.90

2. 60

2.15

1.90

2.00

1.40

2.00

1.90

2.85

1.90

1.90

3.75

1. 40

1.40

1.65

Per Lb.

$\$ 2.75$

2.75

2.75

10 Lbs. and over Per Lb.

$\$ .30$ 
TLRNIPS-Continued

10 Lbs. and

Lb.

Purple Top White Globe...... . . 32

Seven Top or Southern Prize... $\quad .60$

White Egg................. . .35

Yellow Amber Globe........ .40

over Per Lb.

TURNIPS for Stock Feed

Bortfelder Danish (Large Long

Yellow)................ .30

Long White Cowhorn............ .35

.30

.55

.30

.35

SWEDES, or RUTABAGAS

American Purple Top........ . .35

Butter Yellow............ . .40

Purple Top Yellow............. . . 35

White Sweet or Russian. . . . . . . . . 35

Golden Heart (New) . . . . . . . . . .40

HERBS not less than $1 / 4$ pound at

Per Oz.

the pound rate.

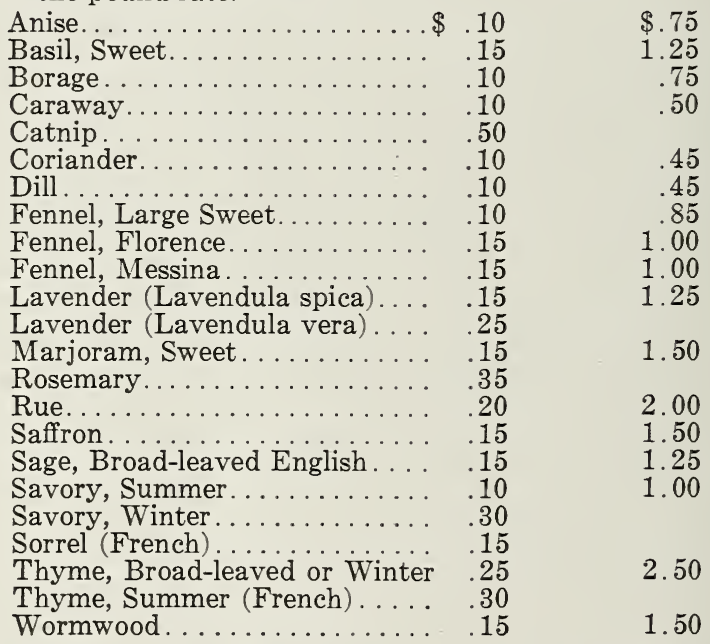

\section{FIELD AND MISCELLANEOUS SEEDS}

$10 \mathrm{lbs}$. or over at $100 \mathrm{lb}$. rate.

Bu. Lb. 100

\section{Barley}

Alpha, pedigreed two-rowed. Developed by plant breeders at Cornell. Out-yields all other varieties, both six-rowed and tworowed, in College tests for past six years. Bearded, stools well, stiff stalk, plump kernel. Ripens with oats. Excellent variety to use with oats and grown for seed. Fields college inspected and seed certified for purity. Bu. of 48

lbs................ $\$ 1.85$

Early Spring. Bu. of $48 \mathrm{lbs} . .1 .75$

Broom Corn, Improved Evergreen

$\$ .09 \$ 8.00$ 
Buckwheat, New Japanese (Bu. of 48 lbs.)............... 1.85

Silver Hull (Bu. of 48 lbs.) . . . 1.75

Cow Peas, New Era (Bu. of 60 lbs.)

Whip-poor-will (Bu. of $60 \mathrm{lbs}$.).

Mixed (Bu. of $60 \mathrm{lbs}$.).

Millet, Golden (Bu. of $48 \mathrm{lbs}$.) ...

Hungarian (Bu. of $48 \mathrm{lbs}$.) .....

Japanese or Billion Dollar Grass

Grass (Bu. of 32 lbs.). . . . . . .

Oats

Swedish or Washington Extra

Heavy (Bu. of 32 lbs.) ....... 1.00

Rape, Dwarf Essex.

Rye, Rosen, the best winter variety (Bu. of $56 \mathrm{lbs}$.) ............. 1.90

Spring (Bu. of 56 lbs.)....... 2.50

Sorghum, Early Amber.........

Sudan Grass, Fancy . . . . . . . . . . .

Soy Beans, Mammoth Yellow

(Bu. of 60 lbs.) ........... 3.40

Medium Early Green (Bu. of 60

lbs.) ................... 4.25

Sunflower, Mammoth Russian...

Hairy or Sand Vetch (Vicia villosa) Winter................

Wheat, Spring (Bu. of $60 \mathrm{lbs}$.) ...

Winter, Rochester, Number Six,

white, beardless (Bu. of $60 \mathrm{lbs}$.). 2.50

Bird Seeds. Canary Sicily...... .

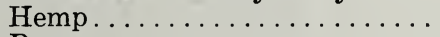

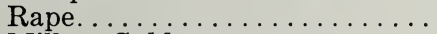

Millet, Golden ...........

Lettuce..................

Mixed Bird Seeds.

$\$ .08 \quad \$ 6.50$

$.07 \quad 5.50$

$.07 \quad 5.50$

$.11 \quad 10.00$

$.08 \quad 6.00$

$.10 \quad 7.50$

\section{CLOVER AND GRASSES}

Kentucky Blue Grass (Poa pratensis), Per Lb. 100 Lb. fancy or double extra clean, for fine lawns. $\$ \quad .42 \quad \$ 40.00$

Red Top (Agrostis vulgaris) Fancy ........ .40 38.00

Crested Dog's Tail (Cynosurus cristatus).. $\quad .55 \quad 50.00$

Orchard Grass (Dacytlis glomerata)..... . . . $25 \quad 23.00$

Sheep's Fescue (Festuca ovina) .......... $\quad .30 \quad 28.00$

Meadow Fescue (Festuca pratensis) . . . . . . . . $23 \quad 21.00$

Red Fescue (Festuca rubra) . . . . . . . . . .28 26.00

Perennial Rye Grass (Lolium perenne) ... $\quad .16 \quad 15.00$

Timothy (Phleum pratense) Per bu. $\$ 4.00 \quad .10 \quad 8.88$

Vick's Velvet Lawn Grass . . . . . . . . . . . .40 37.00

Vick's Exposition Park Mixture . . . . . . .45 40.00

Vick's Shady Spot Lawn Grass . . . . . . . .40 $\quad 37.00$

GLOVER SEEDS, Etc.

White Clover, first quality per lb. $\quad \$ .55^{60}$ lbs. $\$ 50.00$

Medium Red ................. \$21.00 $\quad 35.00$

Mammoth Red .............. $21.50 \quad 36.50$

Alsike..................... $16.50 \quad 27.50$

Alfalfa (Lucerne) . . . . . . . . . . . . $13.00 \quad 21.67$

White Blossom Sweet Clover (biennial). $7.50 \quad 12.50$

Hubam Clover. The new annual sweet clover.................. per lb. $\$ .35$

Ontario Variegated Alfalfa. Our seed

32.00 comes direct from the center of the Alfalfa growing section of Canada where this variety originated............\$14.00 


\section{Choice Flower Seeds VICK'S FAMOUS ASTERS}

\section{Extra Early Varieties}

Queen of the Market. White, Peach Blossom or Pink, Rose, Light Rose, Light Blue, Dark Blue, Scarlet, Lavender, Crim-

son and mixed. White, Purple, Lavender,
Vick's Royal. W.... Lavender Pink, Rose, Shell Pink and

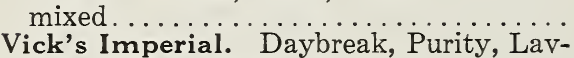
ender, Lilac and mixed............ 1.25

\section{Midseason Varieties}

Early Branching. Shell Pink, White, Rosy Red, Lavender Pink, Rose, Lavender, Crimson, Purple, Dark Violet and mixed. 1.00

Heart of France................. 1.25

Mikado. White, Crimson, Shell Pink, Rose, Dark Violet and mixed........... 1.00

Perfection. White, Shell Pink, Blush, Lavender, Silvery Rose, Rose, Crimson, Purple and mixed.................... 1.25

\section{Main Crop, Late Varieties}

Rochester. Lavender Pink, the original "Rochester," Shell Pink, Lavender, Rose, Cerise, Crimson, Purple, White and mixed 1.25

Vick's Late Branching. Shell Pink (Mary Semple), Lavender (Azure Blue), Lavender Pink, Pale Lavender (Carlson's), Purple, Rose (Lady Roosevelt), Rosy Carmine, Silvery Rose, Scarlet Gem, (Sensation), Dark Violet, Crimson, White and mixed.

Vick's Late Branching (Non-lateral type). Peach Blossom, Rosy Pink or Deep Pink, Lavender, Lavender Pink, Rose and mixed ................... 1.00

Vick's King Asters (Branching Type). Crimson, Violet, White, Shell Pink, Rose, Lavender and mixed.............. 1.25

Vick's King Asters (Upright Type) White, Shell Pink, Rose, Lavender, Lavender Pink, Crimson, Dark Violet and

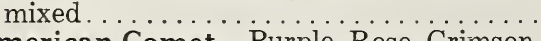

American Comet. Purple, Rose, Crimson, White, Shell Pink, Lavender, Purple and mixed.

Improved Crego. Shell Pink, Rose, Crimson, White, Purple, Lavender and mixed.

Late Upright. White, Shell Pink, Rose, Crimson (Giant Crimson), Amethyst, Lavender, Purple, Rosy Red and mixed. .\$1.00

\section{Mixtures}

All Classes and Colors Mixed..........

Novelty Mixture................ 1.25

ABUTILON

Extra fine varieties mixed........ \$2.25

ACHILLEA (The Pearl) ......... 2.50

ACONITUM Napellus............ 1.15
$\mathrm{Lb}$.

15.00

10.00

$\$ 10.00$

7.00

15.00 
Oz. Lb.

ACROCLINIUM (Everlasting)

$\$ .20 \$ 2.00$

Single Mixed varieties........... $\$ .20$

2.50

ADLUMIA CIRRHOSA (Allegheny Vine).

AGERATUM

1.30

Coeruleum Blue. . . . . . . . . . . . . . .25

Little Dorritt, azure blue........... $\quad .45$

Little Dorritt, white............. .50

Blue Perfection, dwarf, dark blue.... $\quad .40$

Princess Victoria Louise. . . . . . . . . . . . .40

Mixed varieties.............. .25

2.50

4.40

5.00

4.00

4.00

2.50

AGROSTEMMA CORONORIA Crimson.

2.00

\section{AGROSTIS}

Nebulosa, Cloud Grass (Ornamental). .

\section{ALYSSUM}

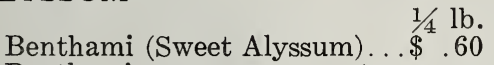

Benthami compactum erectum "Little Gem"........... 1.50

Benthami compactum procumbens "Carpet of Snow".... 1.50

Saxatile compactum "Gold Dust," splendid for edging (Perennial)..... AMARANTHUS

Sunrise, very showy plant. . . . . . . .

Tri-color (Joseph's Coat) . . . . . . . . . .

Caudatus (Love Lies Bleeding) red...

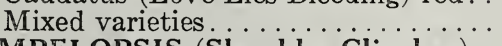

AMPELOPSIS (Shrubby Climber)

Veitchii, Boston or Japan Ivy. $\$ 1 / 4$ lb. ANCHUSA ITALICA

Dropmore.................. 60

ANEMONE (Coronaria) Semi-double 1.50

ANTHEMIS TINCTORIA . . . . . . . . 80

ANTIRRHINUM (Snapdragon)

$1 / 4 \mathrm{Oz}$.

1.65

Fawn, pink and terra cotta shades....

Amber Queen, canary yellow over-laid chamois pink, golden lip...........

Cottage Maid, soft pale pink with white tube......................

Empress, rich velvet crimson........

Gloria, rich chamois . ............

Golden Queen, clear yellow ..........

Purity, pure glistening white, very fine

Silver Pink (special florists' strain)... .

Finest Mixed...................

\section{ANTIRRHINUM MAJUS}

\section{Tall Giant-Flowered}

Venus, shell pink..............

Queen Victoria, white...............

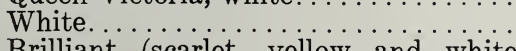

Brilliant (scarlet, yellow and white

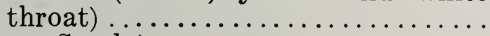

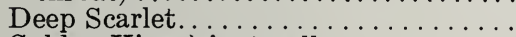

Golden King, giant yellow . . . . . . . .

Roseum, light rose...............

Deep Rose. 
ANTIRRHINUM MAJUS-Cont'd.

$\mathrm{Oz}$.

$1 / 4 \mathrm{Lb}$.

Fire King, orange scarlet with white throat.....................

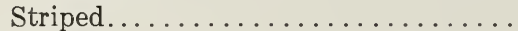

Yellow.......................

Giant-flowered, choicest mixed .......

Tall Varieties, fine mixed regular strain

Dwarf Mixed, extra choice (Tom Thumb)..................

AQUILEGIA (Columbine)

Coerulea, flowers sky-blue and white. .

Vick's Long Spurred Hybrids,

$$
1 / 8 \mathrm{Oz} \text {. }
$$

splendid large flowering strain.............. .50

Chrysantha, golden yellow....

Skinnerii, yellow, with scarlet spurs. . .............

Double, fine mixed . . . . . . .

Single varieties, mixed.

Mrs. Scott Elliott's Hybrids (true)

$\begin{array}{rr}\$ .55 & \$ 1.60 \\ .45 & 1.50 \\ .45 & 1.50 \\ .45 & 1.40 \\ .30 & 1.00 \\ .55 & 1.60\end{array}$

1.40

4.00

ARABIS ALPINA, White Perennial. .

\section{ASPARAGUS}

Sprengeri..................

Plumosus nanus. . . . . . . . . .

$\$ .15$

.30

$\$ 1.00$

\section{ASPERULA}

Azurea setosa, blue or lavender flowers, sweet-scented.................

BACHELOR'S BUTTON (See Centaurea)

BALLOON VINE (Cardiospermum).

\section{BALSAM}

Improved Camellia-flowered

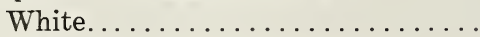

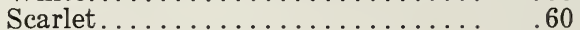

Light Pink. . . . . . . . . . . . . .

Deep Blood Red..............

Camellia-flowered mixed . . . . . . . . .

Fine mixed. A splendid mixture double varieties...............

BALSAM APPLE. See also Gourds...

BALSAM PEAR. See also Gourds....

BEGONIA Erfordia Pink....\$1.50

Vernon, red............ $\$ 1 / 80$

Semperflorens, Alba, white... . .50

BELLIS PERENNIS (See Daisy)

BLUE LACE FLOWER Didiscus Coeruleus...................

BRACHYCOME Swan River Daisy. A beautiful Annual with dainty blue flower................. 
CALCEOLARIA $\frac{1}{32} \mathrm{Oz} . \quad \mathrm{Oz}$.

Lb.

Hybrida grandiflora, large, selfcolored..............\$2.50

Hybrida grandiflora tigrina, finest spotted .......... 3.00

CALENDULA $1 / 4 \mathrm{Lb}$.

Meteor, double yellow, striped with orange.......... .50

Le Proust, Nankeen yellow flushed apricot.......... .60

Double Sulphur. . . . . . . . . .60

Orange King............. .60

Vick's Special Forcing Calendula............... 1.00

$\$ .20 \quad \$ 2.00$

Ball's Strain, bright glowing orange full double, excellent for for cing. . . . . . . . . . .

Mixed varieties, doubles.......

$.20 \quad 2.00$

$.20 \quad 2.00$

$.20 \quad 2.00$

$.25 \quad 2.50$

CALlIOPSIS, Annual Varieties (See also Coreopsis)

Coronata, tall yellow, fine... 1.00

Drummondii, tall (Golden Wave)............... .60

Tall varieties, mixed........ .50

Dwarf varieties, mixed...... .60

\section{CAMPANULA}

2.00

Carpatica, white.............. 1.00

Carpatica, blue.............. 1.00

Persicifolia alba.............. 4.00

Persicifolia coerulea................ 4.00

Pyramidalis Chimney Bell Flower,

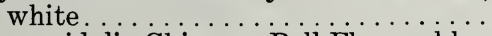

Pyramidalis Chimney Bell Flower, blue

Platycodon Grandiflora, white. .......

Platycodon Grandiflora, blue. ........

Medium, Canterbury Bell, single, rose or pink.................

Medium, Canterbury Bell, single mixed Medium, Canterbury Bell, double mixed.................

Medium, Canterbury Bell, all varieties mixed......................

Calycanthema (Cup and Saucer) mixed

1.00

1.00

1.25

1.25

\section{CAND YTUFT (Iberis)}

Rocket, white, large pyramidal heads. .

Empress, Giant white hyacinth

flowered, very choice...... $1.25 \quad .30$

Carmine................ .75

Flesh-colored . . . . . . . . . .75

Dunnett's Extra Dark Crimson .75

Lilac .................. .75

Pinnata, white, blooms all sum-

\section{CANNA (Indian Shot)}

Crozy's New Hybrids, mixed........ $\quad .20$

Dark Leaved, mixed.............. $\quad .35$

Latest named varieties, mixed........ $\quad .25$ 
$\mathrm{Oz}$.

CARDIOSPERMUM Balloon Vine... \$.10

CARDINAL CLIMBER

1.20

CARNATION (See also Dianthus)

Hardy Perpetual flowering, fragrant. Fine for cutting.

Extra fine double, mixed. . . . . . . . .

Vienna Dwarf, early, extra fine, double mixed.

Grenadin Dwarf, early, double bright

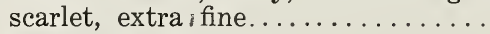

Marguerite, white .............

Marguerite, pure yellow...........

Marguerite, mixed..............

Marguerite, Giant, or Malmaison, mixed, extra large-flowered strain... .

Chaubauds Everblooming............

CASTOR OIL BEANS-See Ricinus

CELOSIA PLUMOSA Feathered

Plumosa superba, beautiful crimson feathered...................

Golden Plume...................

Thompson's feathered (Thompsoni Magnifica)................

Pride of Castle Gould, large brilliant colored tresses...... $\frac{1}{16}$ oz. $\$ .40$

Chinese Woolflower, crimson.........

Feathered Varieties, mixed...........

\section{CELOSIA CRISTATA (Cockscomb)}

Glasgow Prize, fine dwarf soft dark red.

Empress, fine dwarf, dark maroon . . . . .

Dwarf varieties, mixed............

\section{CENTAUREA}

Americana, lilac...............

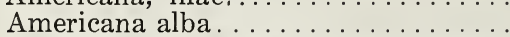

Candidissima, white leaved, dusty miller....................

Gymnocarpa, fine-cut silvery foliage. . .

Cyanus (Bachelor's Button, Blue Bottle, Corn Flower) mixed colors . . . . .

Cyanus, Double, dark blue. ........

Cyanus, Emperor William, dark blue, single....................

Cyanus, Pure White . . . . . . . . . . .

Imperialis (Sweet Sultan) mixed colors.

Odorata, light blue. . . ...........

Margueritae, pure white, very large, sweet-scented, fine for cutting......

Suaveolens Yellow Sweet Sultan......

CHRYSANTHEMUM Annual varieties

Inodorum, Bridal Robe, Double, pure

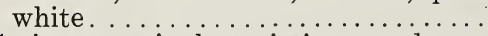

Carinatum, single varieties mixed....

Carinatum, double varieties, mixed...

Coronarium, Double White.........

Coronarium, Double Yellow. 
CLARKIA-Single Mixed

Double Mixed.

CLEMATIS PANICULATA.

COBOEA (Ornamental Climber)

Scandens, blue...............

COIX LACHRYMA-Job's Tears-

Ornamental Grass......... $1 / 4 \mathrm{Lb} .25$

$$
1 / 8 \mathrm{Oz} \text {. }
$$

COLEUS-Finest Mixed . . . . \$ .70

COLUMBINE-See Aquilegia

CONVOLVULUS Morning Glory, see

also I pomoea

Major (Tall Morning Glory) mixed colors, lb. $\$ .75 \ldots . .25$

Minor (Dwarf Morning Glory) mixed colors............. .25

COREOPSIS-Perennial (See also Calliopsis)

Grandiflora, flowers large, our own growing..........\$ .75

CORNFLOWER ASTER-Stokesia Cyanea..................

\section{COSMOS}

Vick's Extra Early Cosmos commences to bloom in July and continues till frost. The finest strain yet produced.

Vick's Extra Early, pure white 1/4 Lb.

Vick's Extra Early, soft pink $\$ 1.25$

Vick's Extra Early, crimson... 1.25

Vick's Extra Early, mixed colors ............... 1.00

Double Crested White Queen . ......

Double Crested Crimson King .........

Double Crested Pink Beauty.........

Double Crested Mixed............

Perfection large-flowered, pink . ......

Perfection large-flowered, white...... .

Perfection large-flowered, crimson....

Perfection large-flowered, mixed $\quad .15$

Lady Lenox, Giant White......... $\quad .25$

Lady Lenox, Giant Pink. . . . . . . . . . . .25

Klondyke, yellow ............ .40

CYPRESS VINE(Ipomoea Quamoclit)

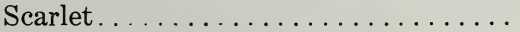

White . . . . . . . . . . . . .

Mixed

DAHLIA

Double Mixed.................

Choicest single varieties mixed......

\section{DAISY (Bellis perennis)}

The Monstrosa varieties are the largest and most vigrous type yet produced.

Monstrosa, rose . . . . . . . . . . . .

Monstrosa, white..............

Montrosa, red ..................

Montrosa, mixed. 
DAISY (Bellis Perennis)-Cont nued

$\mathrm{Oz}$.

Lb.

Snowball, large double, pure white....

1. 60

Longfellow, large double, rosy pink. . .

Mixed, large double, choicest strain. . .

Shasta Daisy, Alaska extra fine stock.

DATURA-Finest mixed, double .

Wrightii, white, tinged with lilac (Fine)

1.60

1. 10

1.20

.25

DELPHINIUM-See Larkspur

DIANTHUS-Pinks (See also Carnation)

Double Annual Varieties

Chinensis, best double, mixed........

Extra fine, double-flowering, mixed....

Double Mourning Cloak, dark red, white margin ..............

Diademetus (Diadem Pink) ..........

Laciniatus, choicest double fringed, mixed.....................

Double Imperial (Imperialis) . . . . . . . .

Single Annual Varieties

Finest single-flowering, mixed ........
Laciniatus, single-flowering fringed, mixed.

DIDISCUS Coeruleus, Blue Lace Flower.................

DIGITALIS (Foxglove) Hardy Gloxiniaeflora

Alba White.....................

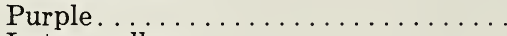

Lutea, yellow . . . . . . . . . .

Rosea, pink...............

Splendid Mixed.

DIMORPHOTHECA

Aurantiaca hybrida, Hybrid African

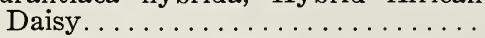

DOLICHOS (Hyacinth Bean) 1/4 Lb.

Lablab, Ornamental Climber, purple............\$ .50

New Japanese. . . . . . . . . .50

DRACENA-Indivisa....... 1.00

White.

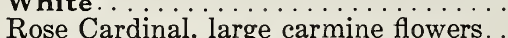

Yellow.....................

Single Orange.................

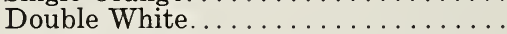

Double Orange. . . . . . . . . . . .

Mikado Orange Crimson . . . . . . . . . .

Single Mixed.................

Double Mixed. . . . . . . . . . . .

\section{EUPHORBIA}

Hetrophylla, Fire on the Mountain (Mexican Fire Plant)............

Variegata, Snow on the Mountain.... $\quad .25$ 
EVERLASTINGS-Continued.

$\mathrm{Oz}$.

Lb.

Gomphrena Globosa Mixed (Globe

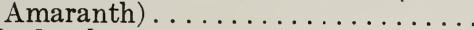

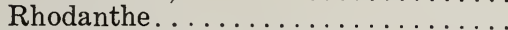

Helichrysum Straw Flower. . . . . . . .

Helichrysum Fire Ball . . . . . . . . . .

Helichrysum Golden Ball. . . . . . . . . . .

Helichrysum Rose Queen. .............

Helichrysum Salmon Queen..........

Helichrysum Silver Ball . . . . . . . . . . .

Helichrysum Violet. ..............

Helichrysum Double Mixed...........

Xeranthenum Double Mixed.

$\$ .25$

$\$ 2.50$

$.40 \quad 4.20$

.45

4.50

.45

4.50

.45

4.50

.45

4.50

.45

.45

.40

4.50

4.50

.40

4.00

4.00

FEVERFEW-See Pyrethrum

FORGET-ME-NOT-See Myosotis

FOUR O'CLOCK - See Marvel of Peru FOXGLOVE-See Digitalis

GAILLARDIA

Picta, single mixed, annual.........

Lorenziana, double mixed, annual. ...

Grandiflora, perennial finest mixed....

GERANIUM (Pelargonium) Zonale,

finest mixed GLADIOLUS-(Hybrid) Mixed, fine assortment. ......................

Finest Mixed ..........\$ .50

GODETIA, choice mixed . . . . . . . .

GOURDS, Ornamental Cucurbitaceae

Dipper or Calabash (Luffa) ..........

Dish Cloth Gourd .................

Echinocystis lobata (Wild $\mathrm{Cu}-$ cumber)........... .60

Egg-formed (True Nest-egg Gourd)...

Hercules' Club. . . . . . . . . . . . .

Momordica balsamina (Balsam Apple)

Momordica charantia (Balsam Pear).

Mixed varieties ......... $\$$ \& .60

GOMPHRENA-(Everlasting)

Globosa, mixed colors............

GRASSES-(Ornamental)

Agrostis Nebulosa-(Annual) Cloud

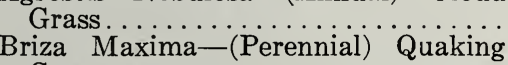

Grass....................

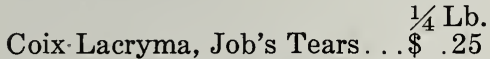

Erianthus Ravennae-(Perennial)

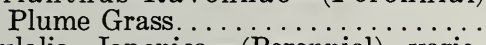

Eulalia Japonica-(Perennial) variegated...................

Pennisetum Longistylum (Annual)

Fountain Grass .................

Pennisetum Ruppelianum (Annual) Purple Fountain Grass. . . . . . . . . . .40

\section{GREVILLEA}

Robusta.

\section{GYPSOPHILA (Baby's Breath)}

Elegans, alba annual, dwarf,

white.............\$ .50 
GYPSOPHILA-Continued.

Oz.

Lb.

Elegans Carmine Annual.....

Paniculata, Perennial white...

$\$ .15$

Paniculata fl. pl. (Dbl.) jwhite. $\$$.. 60

HELIANTHUS (Sunflower) $1 / 4 \mathrm{Lb}$.

Chrysanthemum-flowered, finest of all..........\$ .60

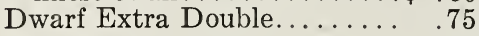

New Red.............. .90

Mammoth Russian.........

HEL I CHRYSUM-Monstrosum (Everlasting or Straw Flower)

Fire Ball, red ................ .45

Silver Ball, white.............. .45

Golden Ball, yellow............. .45

Rose Queen, carmine-rose . . . . . . . . . .45

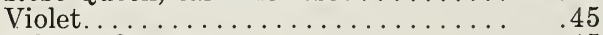

Salmon Queen. . . . . . . . . . . . . . .45

Double Mixed . . . . . . . . . . . . . . 40

\section{HELIOTROPE}

Large-flowering, finest mixed....... 1.50

\section{HOLLYHOCK}

Double ${ }^{r}$ White. . . . . . . . . . . . . . . . . $\quad .90$

Double Pink. . . . . . . . . . . . . . 90

Double Crimson................ $\quad .90$

Double Rose. . . . . . . . . . . . . . . . . .90

Double Yellow ................. $\quad .90$

Double Blood Red.............. $\quad .90$

Double Maroon . . . . . . . . . . . . . $\quad .90$

Double Newport Pink, Enchantress color.

Mammoth Fringed Allegheny, choice mixed colors . . . . . . . . . . . . . . .

Single Mixed. . . . . . . . . . . . . . .

HUMULUS-Japanese Climbing Hop

HUNNEMANNIA (Giant Yellow Tulip Poppy)

Fumariaefolia, fine for cutting.......

\section{HYACINTHUS Candicans,} large spreading heads of white flowers.........\$ .50

HYACINTH Bean-See Dolichos

\section{IBERIS-See Candytuft}

ICE PLANT (Mesembryanthemum)

Useful in porch boxes..........

\section{IPOMOEA}

Bona Nox (white-seeded Moonflower). $\quad .25$

Giant Pink Hybrid Moonflower. . ..... $\quad .30$

Imperial Japanese Morning Glory . . . . . . 20

Morning Glory, Rochester......... . .30

Quamoclit-See Cypress:Vine........

Setosa (Brazilian Morning Glory)....

Cardinal Climber................

IRIS-Kaempferi (Japanese Iris) ... 
JERUSALEM CHERRY, Solanum Oz.

Lb.

capsicastrum nanum ......... $\$ .40$

$\$ 4.50$

JOB'S TEARS-Coix lachryma (Ornamental Grass) $\$ .25 \quad .10$

KENILWORTH IVY (Linaria)

KOCHIA

Tricophylla, True Summer Cypress...............50

Childsii Improved Christmas.. $\quad .60$

KUDZU VINE-Pueraria Thunbergi-

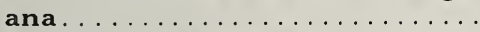

LANTANA -Finest Mixed . . . . . . . .

LARKSPUR, ANNUAL

Double Dwarf Rocket, mixed........

Double Tall Rocket, mixed...........

Double Stock-flowered, mixed..........

LARKSPUR, PERENNIAL-Delphinium

Formosum, dark blue............

Nudicaule, dwarf scarlet. . . . . . . . . . .

Belladonna, best light blue for florists. .

Bellamosum, dark blue type of Belladonna...................

Gold Medal Hybrids, very fine strain. .

Chinese Mixed

LATHYRUS-See Peas perennial

LINARIA (Kenilworth Ivy) . . . . . . LINUM

Grandiflorum rubrum, scarlet fląx, an-

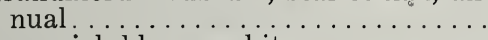

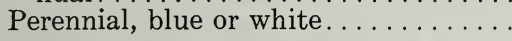

LOBELIA
Cardinalis, perennial (Cardinal
(16 $^{\frac{1}{16}} \mathrm{Oz}$. Flower) brilliant scarlet. ...\$.60

Barnard's Perpetual, deep blue, with white eye, very choice trailing......

Erinus (gracilis) blue 6 to 12 inches...

Erinus, mixed 6 to 12 inch border plant

Erecta compacta, Crystal Palace, dark blue, for edging and carpet bedding.

Erecta Emperor, light blue, very effective................

LOVE-IN-A-MIST-(Nigella) $\$ \frac{1 / 4 \mathrm{Lb} .}{\$} .60$

LUNARIA Honesty (Moonwort) purple or white.

LUPINUS-Polyphyllus Perennial

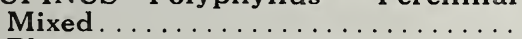

Blue......................

Rose.........................

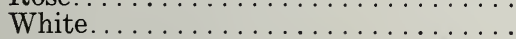

\section{LYCHNIS}

Chalcedonica, bright scarlet.........

Haageana hybrida, fine mixed.........

Viscaria splendens, fine scarlet. . . . . . .

LYTHRUM-Roseum Superbum . . . .

\section{MARIGOLD}

Double African, Lemon Queen........

Double African, Pride of the Garden, dwarf golden, early............ 
MARIGOLD-Continued.

Double African, Prince of Orange.....

Double African, Dwarf Orange.......

Double African, Tall Mixed varieties. .

French Double Striped. ...........

French Gold Margined, dwarf, double.

French Little Brownie, or Legion of Honor, single dwarf, yellow and brown....................

French Dwarf varieties, mixed......

French Tall varieties, mixed.........

Tagetes signata pumila, fine for edgings

MARVEL OF PERU (Four O'Clock or

Mirabilis)
Mixed colors ........... $1 / 4 \mathrm{Lb}$.

MATRICARIA CAPENSIS-See also Pyrethrum

Double White Feverfew Half Hardy Annual...................

MAURANDYA (Fine Ornamental Climber)

Finest mixed

MESEMBRYANTHEMUM-Crystallinum

(Ice Plant)

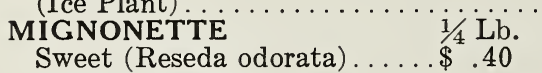

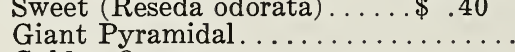

Golden Queen...................

Machet, true, dwarf, finest for pot culture and borders...............

Machet, extra selected stock.........

Allen's Defiance. ...................

Goliath, extra choice, mammoth red spikes......................

White Pearl, best white. . . . . . . . .

MIMOSA

Pudica (Sensitive Plant).............

MIMULUS (Monkey Flower) (Musk Plant)

Hybridus Tigrinus Grandiflorus, prize strain.

$\mathrm{Oz}$.

$\$ .50$

.50

.30

.40

.40

$\$ 6.00$

5.00

3.00

4.00

4.00

.30

3.00

2.50

2.50

.25

.10

1.00

Mixed varieties (all sorts) . . . . . . . .

MIRABILIS-Marvel of Peru

$$
1 / 4 \mathrm{Lb} \text {. }
$$
or Four O'Clock . . . .....\$.40

MOMORDICA-See Gourds

MONKSHOOD-See Aconitum

MOON FLOWER-See Ipomoea

MORNING GLORY-See Convolvulus and Ipomoea

MOURNING BRIDE-See Scabiosa

MUSK PLANT-See Mimulus

MYOSOTIS (FORGET-ME-NOT)

Alpestris Blue................

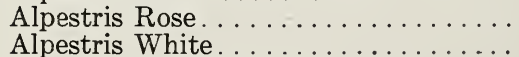

Alpestris White $\ldots \ldots \ldots \ldots \ldots \ldots \ldots$

Robusta Grandiflora (Eliza Fonrobert).

Victoria, sky blue, for pots..........

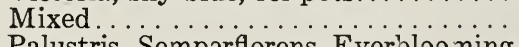

Palustris Semperflorens Everblooning Blue.................... 2.00

Dissitiflora Perfection, extra $\frac{1}{16} \mathrm{Oz}$. large............. $\$^{16} .30$

8.00

8.00

8.00 
NASTURTIUM, DWARF or Tom Thumb

Any of the following dwarf varieties $\$ .15$ per oz., Mixed $\$ .10$ per oz.

Aurora, Salmon-rose, mottled and veined..................

Beauty, orange and vermilion......

Bronze-colored, distinct. ...........

Carters Scarlet Atrococcineum.......

Chamaeleon, variegated...........

Cloth of Gold, golden foliage.........

Coerulea rosea ...................

Crystal Palace Gem, Sulphur, Maroon

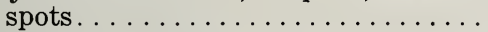

Dark Crimson . . . . . . . . . . . . . . .

Empress of India, dark leaves and very dark crimson flowers. . . . . . . . . .

Golden King. . . . . . . . . . . . . . . .

Golden Queen, yellow foliage. . . . . . .

King Theodore, very dark..........

King of Tom Thumbs, brilliant scarlet

Lady Bird Golden Yellow, flamed crim-

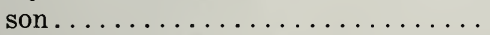

Pearl, light cream color...........

Prince Henry, marbled yellow and scarlet.....................

1/4 Lb. Lb

$\$ .35 \quad \$ 1.00$

$.35 \quad 1.00$

$.35 \quad 1.00$

$.35 \quad 1.00$

$\begin{array}{ll}.35 & 1.00\end{array}$

$.35 \quad 1.00$

$.35 \quad 1.00$

$.35 \quad 1.00$

$.35 \quad 1.00$

$\begin{array}{ll}.35 & 1.00\end{array}$

$.35 \quad 1.00$

$\begin{array}{ll}.35 & 1.00\end{array}$

$\begin{array}{ll}.35 & 1.00\end{array}$

$.35 \quad 1.00$

$\begin{array}{ll}.35 & 1.00\end{array}$

$.35 \quad 1.00$

Ruby King, foliage very dark. . . . . . .35 $\quad 1.00$

Regaleanum, crimson........... . . $35 \quad 1.00$

Spotted King . . . . . . . . . . . . . $35 \quad 1.00$

Vesuvius, rose ................ .35 1.00

Yellow (Luteum) ............. $\quad .35 \quad 1.00$

Vick's Special Mixture from choicest
varieties................... .25

Mixed varieties....... $10 \mathrm{lbs} . \$ 5.50 \% \quad .20 \quad .60$

\section{NASTURTIUM, tall or climbing}

Any of the following varieties $\$ .10$ per oz.

Atropurpureum, dark crimson.......

Coccineum, bright scarlet..........

Dunnett's Orange (Sunlight)........

Edward Otto, bronze, silky and glitter-

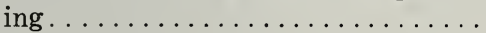

Chocolate. . . . . . . . . . . . . . . .

Jupiter, large golden yellow. . . . . . . . .

King: Theodore, maroon flowers, dark foliage. . . . . . . . . . . . .

Luteum, pale yellow ...............

Prince Henry, marbled yellow and

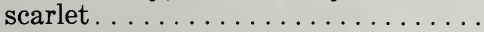

Pearl (Moonlight) creamy white.....

Regelianum, purple-violet. .........

Scheuerianum, straw-colored, spotted .

Schillingii, yellow, brown spotted.....

Schultzii, brilliant scarlet, dark leaved

Vesuvius, brilliant salmon-rose.......

Von Moltke, blush rose............

Fine Mixed ........10 lbs. $\$ 4.50$

Extra Choice Mixture 
NASTURTIUM, Climbing, Continued-1/4 Lb. $\mathrm{Lb.}$

NASTURTIUM Lobb's Tall

Hybrids of Madame Gunther, splendid

mixed.

NASTURTIUM Ivy-leaved varieties

$\mathrm{Oz}$.

Dwarf Mixed............\$.15

Tall Mixed....................... 15

NASTURTIUM-Canariense, Canary

$\mathrm{Oz}$.

Bird Flower.

NEMOPHILA Baby's Eyes, mixed...

NICOTIANA

Affinis, large white flowers, very frag-

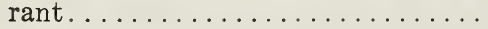

Affinis hybrids. . . . . . . . . . . .

Sanderae, finest strain .

NIGELLA (Love-In-A-Mist)

Damascena, double mixed .............
Miss Jekyll, double blue, very

fine............ $1 / 4$ Lb. $\$ .60 \quad .20 \quad 2.00$

\section{PANSIES, GIANT FLOWERING}

Our strains of this important flower have been selected especially for the most critical florists use. Every variety and all mixtures are always grown in our trial grounds and only those that come up to true Vick Quality are ever catalogued.

\section{GIANT STRAIN in Separate Colors}

Oz. $\quad 1 / 4 \mathrm{Lb}$.

Adonis, light blue. $\$ 1.25$

Emperor, blue, purple eye........ 1.25

Fire King, golden yellow purple edge.

Goldelse, pure yellow............

King of the Blacks, extra fine........

Lord Beaconsfield, purple-violet, shading to, white..................

Prince Bismarck, light brown shades, with dark eye, fine............

Ruby King, Red Shades.............

Snowflake, extra fine pure white.....

Yellow, with dark eye............

White, with purple eye..............

New Orange Pansy. (St. Knud) an entirely new, distinct color in a pansy, fine large flowers.

1.25

$1.25-4.00$

$1.25 \quad 4.00$

$1.25 \quad 4.00$

$1.25 \quad 4.00$

$1.25 \quad 4.00$

$1.25 \quad 4.00$

$1.25 \quad 4.00$

$1.25 \quad 4.00$

$1.50 \quad 5.00$

\section{PANSIES-Special mixtures}

Vick's Giant Superb Mixed. The very finest strains obtainable from the best pansy specialists at home and aboard. Flowers of immense size perfect form and wonderful combinations of color. $\frac{1}{32}$ oz., $\$ .50, \frac{1}{16}$ oz. $\$ .85,1 / 8$ oz. $\$ 1.50,1 / 4$ oz. $\$ 2.50$, oz. $\$ 8.00$.

Bugnot's, superb blotched mixed........... $\frac{1}{16} \mathrm{Oz}$ \$.30

Madame Perret-Rose and Crimson shades.................

Masterpiece, new class of Giant $\frac{1}{16} \mathrm{Oz}$.

varieties, fine rich colors, giant flowers with waved or frilled edges............... .40

Vick's Peerless Mixed . . . . . . . . 30 
PANSIES, Giant-flowering (Con't) Oz.

Mastodon Mixed. A new strain of American Pansies of immense size, splendid form, beautiful colors........\$ .50 4.20

Trimardeau, Giant's mixed . . . . . . . 1.60

Standard varieties mixed.......... $\quad .90$

PANSY VIOLET (Viola cornuta (Tufted Pansy)

Fine strain of our own growing. Perfectly hardy.

Blue Perfection .............. 2.00

Perfection White................ 2.00

Yellow..................... 2.00

Mixed ..................... 1.50

PAPAVER-See Poppy

PEAS, PERENNIAL Lathyrus (Ever- Oz. Lb. lasting Pea)

Latifolius, white.............. .75

Latifolius, rose................ $\quad .75$

Latifolius, crimson................ $\quad .75$

Mixed................... . 50

PENNISETUM (Fountain Grass) See Grasses

PENTSTEMON

Barbatus (Perennial Red) ......... 2.00

PERILLA (Beautiful Annual foliage plant)

Nankinensis-Foliage bronze purple..

\section{PETUNIA}

Single Bedding Varieties

Belle Etoile, striped and blotched....

Countess of Ellesmere, dark rose white throat.

General Dodds, dark red.............

Howards Star, dark red with distinct white star . . . . . . . . . . . . . .

Rose of Heaven, brilliant rose.......

Rosy Morn, soft pink. ...........

Snowball, pure white............

Mixed

1.20

1.60

1.60

1.60

1.50

.60

1.00

PHLOX DRUMMONDII-One of our specialties

\section{Grandiflora Varieties}

Alba pura, pure white............

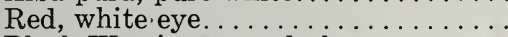

Black Warrior, ${ }^{\circ}$ very dark maroon . . . .

Brilliant, , deep pink shaded lighter at edges....................

Chamoist Rose. salmon pink.........

Isabellina (lutea) yellow...........

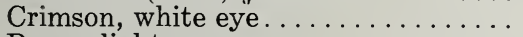

Rosea, light rose. . . . . . . . . . . . .

Rose, white eye. . . . . . . . . . . . .

Splendens, brilliant scarlet.

Extra Choice Mixed. This stock is extra fine, being a well-balanced mixture of the

most brilliant colors....... 
PHLOX DRUMMONDII-Continued .

Oz. Lb.

Dwarf Varieties-Nana Compacta

Fireball, fiery scarlet........... \$2.00

Snowball, pure white.......... 2.00

Best dwarf varieties mixed......... 1.25

PHYSALIS FRANCHETTI Chinese

Lantern Plant........... .75

PHYSOSTEGIA-Virginica........ 1.00

PINK-See Dianthus

PLATYCODON-See Campanula

POPPY

California, see Eschscholtzia

Single Annual Varieties $\quad 1 / 4 \mathrm{Lb}$.

Danebrog (Danish Flag) white cross on scarlet ground.....\$ .50

Miss Sherwood, satiny white and rose................ 45

Shirley, extra fine, our own growing ............ 60

Double Annual Varieties

Carnation-flowered mixed .... .50

Cardinal, new hybrid, in splendid mixture.....................

Mikado, white and scarlet striped....

Peony-flowered mixed..............

Ranunculus flowered, dwarf.........

\section{Perennial Varieties}

Excelsior Hybrids of the Oriental Poppy. The result of several years' careful selection. Ranges from the most delicate salmon to deep crimson

Oriental, darkest red, purple spots....

Iceland (Nudicaule) yellow . . . . . . . . .

Iceland (Nudicaule white...........

Iceland (Nudicaule) orange..........

Iceland (Nudicaule) mixed...........

PORTULACA

Grandiflora single, finest mixed.......

Grandiflora double, choicest of all mixtures..................... 1.75

PUERARIA Thunbergiana

(Kudzu Vine)

PYRETHRUM

Hybridum, single mixed. . . . . . . . 1.50

Hybridum, double mixed........ 4.00

Double White Feverf-w (Matricaria capensis) ........

Aurea Golden Feather (yellow foliage). $\quad .35$

RICINUS (Castor Oil Beans)

Borboniensis, green, very large.......

Cambodgensis, leaves and stems nearly

Sanguineus, red .................... $\quad .15$

Zanzibariensis, in finest mixture..... . .15

Mixed varieties.............. $\quad .10$

RHODANTHE (Everlasting) mixed... $\quad .40$ 
SALPIGLOSSIS (Velvet Trumpet)

$\mathrm{Oz}$.

Lb.

Very easily grown annual. A wonderful strain, large flowers, rich velvety colors. One of our specialties.

Crimson and gold . . . . . . . . . . \$1.00

Light blue and gold............. 1.00

Velvety red................. 1.00

Scarlet and gold.............. 1.00

Violet...................... 1.00

Yellow .................... 1.00

Purple, red and gold............ 1.00

Emperor, finest mixed............. $\quad .70$

Choice mixed ............... .50

SALVIA

America, Globe of Fire, new dwarf... 3.50

Patens, splendid sky blue.... $\$^{\frac{1}{16}} .70$

Splendens, true, large-flowered, scarlet 1.20

Bonfire (Clara Bedman)........... 2.00

Zurich, early dwarf ............. 3.50

\section{SCABIOSA (Mourning Bride)}

Black purple.............. $\quad .45$

Double tall white (Snowball) ....... $\quad .45$

Double yellow, tall. . . . . . . . . . . .45

Double Lilac, tall . . . . . . . . . . . . . .45

Double fiery scarlet............ .45

Double tall, mixed.............. .30

Double dwarf, mixed. . . . . . . . . . $\quad .30$

Japonica, perennial. ........... . .50

Caucasica Lilac Perennial . . . . . . . . . 3.00

\section{SCHIZANTHUS}

Wisetonensis, splendid pot plant..... $\quad .80$

SENSITIVE PLANT Mimosa pudica. $\quad .30$

SHASTA DAISY Alaska........... 1.20

SMILAX BOSTON Myrsiphyllum asparagoides............... .30

SNAPDRAGON-See Antirrhinum

SOLANUM

Capsicastrum Nanum, Jerusalem Cherry..................

Cleveland Cherry, more compact in growth than the old Jerusalem Cherry and bears more berries.

STATICE, Sinuata (Everlasting)

Blue.......................60

Lavender......................... $\quad .60$

Rose.............................6

White..................... .60

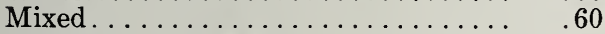

STOCKS

Stocks are one of the most profitable flowers to grow, especially if you have the right seed. We offer only proved stock of the highest quality, having the smallest percentage of singles.

Large Flowering, Ten Weeks

This type is used mostly for outdoor growing for summer blooms.

Azure blue...................... 1.80

Canary yellow . . . . . . . . . 1.80 
STOCKS-Ten Weeks-Continued

Bright pink................ $\$ 1.80$

White................... 1.80

Blood red.................... 1.80

All colors mixed. . . . . . . . . . . . . . 1.20

Wallflower leaved mixed.......... 1.80

"Nice" Stock, Early Large Flowering The finest strain for early forcing. Giant flowering

Flesh pink...................

Giant white. . . . . . . . . . . .

Giant yellow.................

Giant crimson..................

Queen Alexandra (rosy lilac)........

Empress Elizabeth, carmine rose.....

Mixed......................

Perpetual Branching (Cut and Come Again)

Remarkable for its branching habit. Strong and vigorous

Empress Augusta Victoria, lilac. . . . . .

Princess Alice (white) . . . . . . . . . . .

Rose... . . . . . . . . . . . . . . . . .

Blue......................

Blood red...................

Yellow.......................

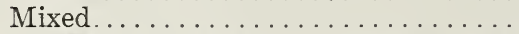

STOKESIA Cyanea (Cornflower Aster $\ldots \ldots \ldots \ldots \ldots \ldots \ldots \ldots$

SUNFLOWER-See Helianthus

SWAN RIVER DAISY-See Brachycome

\section{SWEET PEAS}

\section{Summer Flowering Spencer Varieties}

Agricola Spencer. White flushed with

Oz. $1 / 4 \mathrm{Lb}$. Lb. heliotrope..................\$.20

America. Striped red and white..... . 20

$\$ .50 \$ 1.40$

Asta Ohn, Spencer. Light lavender.

Cherub. Rich cream edged with deep rose.....................

Constance Hinton. Large white, black seeded....................

Countess Spencer. Rich pink, deeper

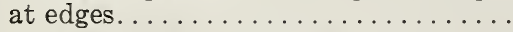

Crimson King. Fine new crimson....

Dainty Spencer. White with delicate

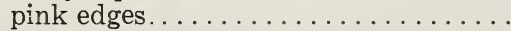

Daisybud. Apple bloosom tints and

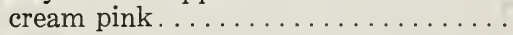

Defiance. New orange scarlet...... 1.00

Dobbie's Cream. Primrose.......... .20

Elfrida Pearson. Mammoth light pink

Florence Morse. Clear light pink....

Geo. Herbert. Rich rose-crimson..... .20

Hawlmark Pink. Fine new rose pink . .20

.501 .40

$.20 \quad .50 \quad 1.50$

$.20 \quad .50 \quad 1.50$

$.20 \quad .50 \quad 1.50$

.20

$.50 \quad 1.50$

$.80 \quad 2.50$

$\begin{array}{lll}.20 & .50 & 1.50\end{array}$

$\begin{array}{lll}.20 & .50 & 1.50\end{array}$

$3.00 \quad 8.00$

$.50 \quad 1.50$

.501 .50

$.50 \quad 1.40$

$.50 \quad 1.40$

.501 .60

Hebe. Fine rich pink with broad standards 


\section{SPENCER SWEET PEAS}

Continued

Hercules. A large-flowering Countess Spencer.................... \$.20 \$.50 $\$ 1.40$

Illuminator. Gorgeous orange salmon. $\quad .20 \quad .50 \quad 1.40$

King Edward, Spencer. Crimson-scar-

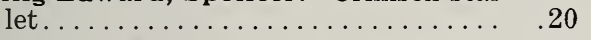

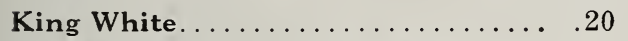

$.50 \quad 1.40$

$.50 \quad 1.50$

$\begin{array}{lllll}\text { Majestic Cream. Rich deep cream.... } & .20 & .50 & 1.50\end{array}$

Margaret Atlee. Pink cream, suffused with salmon ................ .20 .50 1.50

$\begin{array}{lllll}\text { Margaret Madison. Clear azure blue.. } & .20 & .50 & 1.40\end{array}$

Mrs. Cuthbertson. Standard rose-pink

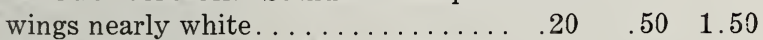

Mrs. Hugh Dickson. Mammoth apricot and buff ................20 .20 .50 1.40

Mrs. C. P. Tomlin. A new fiery scarlet. $\quad .20 \quad .50 \quad 1.50$

Mrs. Tom Jones. A new blue, the finest

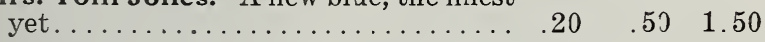

Picture. One of the largest cream and

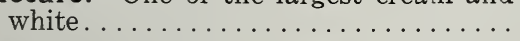

Renown. Splendid new rose carmine.

R. F. Felton. Rich lavender pink.....

Rosabelle. Fine large rose-colored flower

Royal Purple. Large flower, rich purple

Royal Scott. New brilliant scarlet or

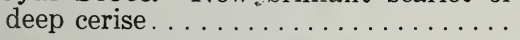

Sunset. Splendid novelty, soft rich rose,

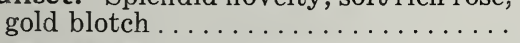

The President. Brilliant scarlet..... $.20 \quad .50 \quad 1.50$

Thos. Stevenson. Orange scarlet.... $\quad .20 \quad .50 \quad 1.50$

Warrior. Reddish maroon with bronze tints ........................ .20 .50 1.50

$\begin{array}{llll}\text { White Spencer. Splendid pure white. . } & .20 & .50 & 1.50\end{array}$

Wedgewood. A good blue.......... .20 $.50 \quad 1.40$

Spencer mixed extra choice........... $.15 \quad .40 \quad 1.25$

Spencer fine mixed ................ $10 \quad .30 \quad .90$

Cupid Dwarf, mixed ............... . . .40 15.25

\section{SWEET PEAS}

The Best Grandiflora Varieties

Aurora. White flaked orange-salmon, hooded ..................

Dorothy Eckford. Large, pure white.. Hon. Mrs. E. Kenyon. Primrose fine. Janet Scott. Rich deep pink.........

King Edward VII. A large bright red or crimson-scarlet self . . . . . . . . ......

Lady Grisel Hamilton. Lavender....

Lovely. Shell-pink, extra fine flower...

Lord Nelson. Dark blue............

Miss Willmott. Large orange pink...

Othello. Dark glossy maroon, extra fine

Prima Donna. Soft clear pink.......

Vick's Invincible Mixture..........

$\begin{array}{ll}.25 & .80 \\ .25 & .80 \\ .25 & .80 \\ .25 & .80 \\ & \\ .25 & .80 \\ .25 & .80 \\ .25 & .80 \\ .25 & .80 \\ .25 & .80 \\ .25 & .80 \\ .25 & .80 \\ .20 & .50\end{array}$




\section{SPENCER SWEET PEAS}

Early or Winter Flowering

Oz. Lb. Lb.

All White. Largest waved pure white. $\$ .35 \$ 1.10 \$ 3.50$

Asta Ohn. Fine lavender, same form as late-flowering Asta Ohn...........

Aviator. Dazzling crimson scarlet, flower extra large................

Blanche Ferry. Pink and white, standards bright crimson, wings white tinted

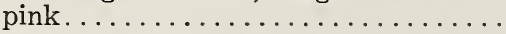

Blue Bird. Violet blue, a charming shade

Cream. A primrose Spencer of fine shape and size, similar to late-flowering Dobbie's Cream..................

Glitters. The sensational new orange scarlet. . . . . . . . . . . . . .

Heather Bell. Rich lavender mauve, flowers are very large, borne in threes and fours on long stems............

Mrs. A. A. Skatch. Clear pink, one of the best of the pink Spencers . . . . . . .

Melody. Rose pink on white ground...

Morning Star. Orange scarlet, flame color standard with orange pink wings

Rose Queen. Unusually long stems, large flowered rose pink...........

Snow Flake. Magnificent early white, a superb form, three or four flowers on

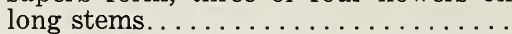

Song Bird. Pale rose pink with white ground, flowers profusely, long stems. .

Spring Maid. Crimson pink, a very beautifully shaded flower, strong grower, long stem, prolific..........

Yarrawa. Best Spencer form, bright rose pink with light wings. ..........

Early or Winter Flowering Mixed...

SWEET SULTAN-See Centaurea

SWEET WILLIAM

Holborn Glory, our own growing, extra fine stock, extra large flowers, auricula eyed.

$\begin{array}{lll}35 & 1.10 & 3.50\end{array}$

$\begin{array}{lll}.35 & 1.10 & 3.59\end{array}$

$\begin{array}{lll}.35 & 1.10 & 3.50\end{array}$

$\begin{array}{lll}.35 & 1.10 & 3.50\end{array}$

$.35=1.10 \quad 3.50$

$\begin{array}{lll}.35 & 1.10 & 3.50\end{array}$

$.35 \quad 1.10 \quad 3.50$

$\begin{array}{lll}.30 & 1.00 & 3.00\end{array}$

$.351 .10 \quad 3.50$

$\begin{array}{lll}.35 & 1.10 & 3.50\end{array}$

$\begin{array}{lll}.35 & 1.10 & 3.50\end{array}$

$\begin{array}{lll}.30 & 1.00 \quad 3.00\end{array}$

$\begin{array}{lll}.35 & 1.10 \quad 3.50\end{array}$

$\begin{array}{lll}.35 & 1.10 \quad 3.50\end{array}$

$\begin{array}{lll}.30 & 1.00 & 3.00\end{array}$

$\begin{array}{lll}.25 & .80 & 2.50\end{array}$

Oz. Lb.

Dunnett's Dark Crimson semi-double

Double White...................

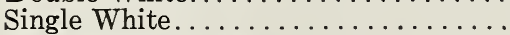

Pink Beauty Single.............

Scarlet Beauty Single. . . . . . . . . .

Double, finest mixed. . . . . . . . . . .

Single, finest mixed ..............

Annual varieties mixed............. 1.00

TAGETES-See Marigold

THERMOPSIS

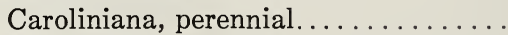

THUNBERGIA.

Alba......................... .50

Rubra................. 40 
VERBENA HYBRIDA

$\mathrm{Oz}$.

Lb.

Mammoth Auricula Flowered....... \$1.00

Mammoth Pure White............ 1.00

Mammoth Scarlet Defiance........ 1.00

Mammoth Blue................ 1.00

Mammoth Pink.............. 1.00

Mammoth Striped............. 1.00

Mammoth Purplish-violet. . . . . . . . 1.00

Mammoth Yellow ............... 1.20

Mammoth Mixed, all colors in finest mixture................... 80

Extra fine mixed............. $\quad .60$

VERONICA (Speedwell) Spicata blue. 1.00

VINCA

Alba pura, pure white......... 1.00

Delicata, soft pink............ 1.20

Rosea, rose................ 1.00

Rosea Alba, white with rose center . . 1.00

Mixed colors.................. $\quad .75$

VIOLA Cornuta-See Pansies Tufted WALLFLOWER

Finest double, tall sorts, mixed...... 2.50

Finest single annual varieties mixed... $\quad .20$

WILD CUCUMBER, Echino$1 / 4 \mathrm{Lb}$.

cystis lobata.........\$.60

XERANTHEMUM Double Mixed

(Everlasting)................40

We take special pride in our strain of Giant Flowering Double Zinnias. The flowers are full double and the separate colors come true to name.

Giant, crimson . . . . . . . . . . . .

Giant, orange. . . . . . . . . . . . .

Giant, purple. . . . . . . . .

Giant, rose. . . . . . . . . . . . . . .

Giant, scarlet. ...............

Giant, striped. . . . . . . . . . . . . .

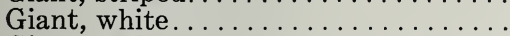

Giant, yellow ............

Giant, mixed ......... \$1.50

Curled and Crested, finest mixed.... .

Lilliput, small very double flowers, charming class for bouquets, mixed colors

New Dahlia Flowered Zinnia. The largest and most gorgeously colored Zinnia grown. Flowers resemble an immense dahlia and the shading of colors is unusually attractive.

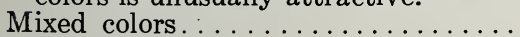

Cactus Flowered.................

The above is not our complete list of flower seeds. If interested in anything not found here write us. We specialize in the finest quality flower seeds of all kinds, 


\section{GLADIOLI}

Per 100

Per 1000

America. One of the most popuGladioli yet introduced. Splendid flowers of a beautiful, clear, flesh pink color............. $\$ 2.40$

Baron Hulot. Violet blue. Dark and velvety. Best of the blues.

Chicago White. Well known and very popular white, lavender markings in throat. Early.....

Empress of India. Rich dark red with darker shadings, almost black..................

Evelyn Kirtland. Rosy pink shading to shell pink in center with scarlet blotches on lower petals..................

Golden West. Rich orange red. Very handsome. . . . . . . . . . .

Gretchen Zang. A beautiful shade of soft pink, blending to scarlet on lower petals. Large flowers

Halley. Delicate rose, with white blotch, extra early and very popular. . . . . . . . . . . . . . . . .

Herada. Large blooms of pure mauve or lavender with deeper markings in throat. . . . . . . . . . .

Kunderdi Glory. The first of the ruffled Gladioli. Cream pink with petals distinctly ruffled at the edge

Le Marechal Foch. New soft pink of enormous size.........

Lily White. One of the best white varieties...................

Loveliness. Creamy white flowers of good form on large broad spikes...................

Mrs. Francis King. Extremely vigorous, long spikes, bright scarlet, a very pleasing shade...

Mrs. Frank Pendleton. Very large. Salmon-pink with carmine blotched throat. .........

Mrs. Watt. Clear wine red, rich velvety texture..............

Niagara. Immense flowers of soft primrose yellow, slightly tinted rose, throat splashed with carmine. ...................

Panama. Seedling of America, which it resembles closely except that it is a deeper shade of pink.

Peace. Beautiful white with pale lilac pencillings on interior petals

Prince of Wales. Delicate coral pink with slight orange shade...

Red Canna. A very good large flowered red............... 
GLADIOLI - Continued.

Per 100

Per 1000

Schwaben. Sulphur yellow, dark blotch in center.............

War. Deep blood red, tall and vigorous..................

Yellow Hammer. Pure yellow with small red mark in the throat.................

$3.25 \quad 27.50$

$4.90 \quad 44.00$

$4.20 \quad 38.00$ Vick's Named Mixtures. This
is a mixture of over 150 named varieties to which we are adding every year. It comprises every class, and will furnish a profusion of bloom from July until frost. Many of these varieties cost us several times as much as we charge for the mixed.........

Choice Mixed. All colors in fine

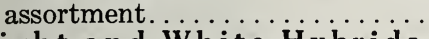

$2.50 \quad 20.00$

$2.00 \quad 17.50$

$2.75 \quad 22.50$

$2.50 \quad 20.00$ grand mixture........... $2.75 \quad 22.50$

Beautiful collection of light and dainty shades, very fine for florists' use..................

Red Hybrids. All shades of red, scarlet and crimson...........

Mixed Hybrids. All shades in

\section{VICK'S DAHLIAS}

Not less than 6 at the dozen rate; 25 at 100 rate

\section{Cactus Varieties}

Cactus Dahlias have large, loosely formed flowers. The long, narrow petals are variously rolled or twisted. The flowers are very graceful and artistic.

Countess of Lonsdale. Rich salmon,

$$
\begin{array}{cc}
\text { Per } & \text { Per } \\
\text { Doz. } & 100
\end{array}
$$
tinted apricot. Hardy and one of the most free flowering of the Cactus type...\$2.75 $\$ 22.00$

Crystal. Clear soft pink. Fine exhibition flower.................... $3.50 \quad 25.00$

Kriemhilde. Exquisite combination of ivory white and pink. "Queen of the Cactus Dahlias". ................. 2.00

J. H. Jackson. Largest and best dark red. 2.00 15.00

General Butler. Deep crimson maroon, tipped white................2.00 15.00

Golden Eagle. Yellow, slightly tinted fawn $3.50 \quad 25.00$

\section{SHOW DAHLIAS}

The old-fashioned double Dahlia. Ball-shaped flowers; the petals quilled or tubular, regularly placed with long stiff stems. Keep well as cut flowers.

Miller's Red. Dark crimson scarlet.

Per Per

$$
\text { Doz. } \quad 100
$$

Large and free flowering...........\$1.40 \$10.00

Stradella. Deep wine rose. Free flowering $1.40 \quad 10.00$

Vivian. White edged rose-violet. Very handsome.................. $1.50 \quad 12.00$ 
SHOW DAHLIAS-Continued.

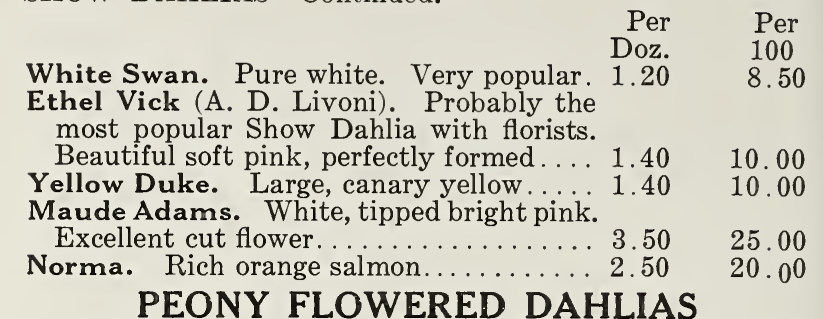

This type is becoming more and more popular. Flowers are semi-double with broad, flat, loosely-formed petals, and golden yellow center. Very graceful.

Dr. Peary. Rich dark mahogany. One of $\begin{array}{cc}\text { Per } & \text { Per } \\ \text { Doz. } & 100\end{array}$

the best dark Dahlias............\$5.00 \$37.50

Hampton Court. Bright mauve pink. A bold flower of splendid form........2.00 15.00

Queen Wilhelmina. Best pure white Peony Flowered Dahlia.............2.00 15.00

Satisfaction. One of the largest Peony type. Clear cream yellow. Long stems. 2.75 20.00

\section{Decorative Varieties}

Intermediate between Cactus and Show. The massive flowers are not so globular as the Show, and the petals are broad and somewhat flattened.

Sylvia or Dolly. White, heavily edged pink. Florists' favorite...........\$1.20 \$8.50

Jack Rose. Rich velvety crimson, early and free flowering. One of the most satisfactory of all................. $1.50 \quad 8.50$

Mina Burgle. One of the finest of the newer decorative varieties. Very large brilliant ruby red ..............2.50 20.00

Princess Juliana. Pure white. One of the best for cutting................ $1.50 \quad 12.00$

Souvenir de Gustav Doazon. Largest Dahlia in existence. Pleasing shade of orange red ................... $1.50 \quad 12.00$

Golden West. Large pure yellow. Long stems........................ $3.50 \quad 25.00$

Delice. Color-bright rose pink. . . . . . 2.50 20.00

\section{POMPOM DAHLIAS}

Ideal for bouquets, being small and very double. Very free flowering.

\section{Per Per \\ Doz. $\quad 100$}

Alewine. White overlaid delicate pink... \$1.50 $\$ 12.00$

Amber Queen. Clear amber, shaded apri-

cot.................... $1.50 \quad 12.00$

Catherine. Clear yellow. Very free.... 1.50 12.00

Darkness. Soft dark velvety maroon. . . 1.50 12.00

Fashion. Orange, perfectly ball shaped. . 1.50 12.00

Snow Clad. White, very free flowering... $1.50 \quad 12.00$

Fine Mixture. All Classes Leading

Named Varieties................ $1.00 \quad 8.00$ 


\section{HARDY PLANTS, BULBS AND ROOTS}

Per Per

Doz. $\quad 100$

Achillea, The Pearl and Millefolium roseum $\$ 1.50$

$\$ 10.00$

10.00

Anchusa Italica, Dropmore Variety. One of the best blue-flowered hardy plants in cultivation................... 1.50

Alyssum, saxatile compactum........ 1.25

Aegopodium Podagraria variegatum.... . 1.25

Agrostemma Flos-Jovis............. 1.25

Anemone, Whirlwind, Queen Charlotte and Japonica alba....................2 2.00

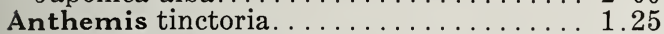

Aquilegia, six varieties........... 1.50

Arabis alpina .............. 1.50

Armeria splendens (Thrift) . . . . . . . 1.25

Asters, assorted (Michaelmas Daisy)..... 1.25

Boltonia asteroides; Latisquama ..... . . . . 1.25

Campanula, persicifolia, persicifolia alba, Canterbury Bell, Carpatica Blue and White....................... 1.25

Campanula Pyramidalis, White and Blue. 1.25

Campanula, Medium............. 1.25

Campanula, Medium Enchantress (new pink) ....................... 1.50

Cerastium tomentosum . . . . . . . . 1.25

Chives...................... 1.25

Clematis Paniculata, $21 / 2$-inch pots . . . . .75

Coreopsis lanceolata grandiflora . . . . . . 1.25

Delphinium Belladonna, Formosum, Formosum Collestinum, Bee Larkspur, Chinese, per $1,000, \$ 95.00 \ldots \ldots \ldots \ldots \ldots 1.50$

Digitalis (Foxglove) Pink, White and Purple 1.25

Erianthus Ravennae............... 1.25

Eulalia Japonica variegata, gracillima univittata and zebrina.............. 1.25

Funkia Japonica, light blue with narrow foliage (Blue Day Lily) . . . . . . . . . . . 1.75

Funkia Subcordata grandiflora (White Day Lily) . . . . . . . . . . . . . . . . 2.50

Funkia Undulata media picta (Variegated Leaved Day Lily).................. 1.75

Gypsophila paniculata............ 1.50

Helenium autumnale superbum, golden yellow flowers, late summer and fall, 5 to 6 feet....................... 1.50

Helianthus, lataefrons, Maximilliana, Multiflorus plenus and ridigus.......... 1.50

Helianthemum vulgare, suitable for border or rockery.................. 1.50

Hemerocallis, flava, Yellow Day Lily (Lemon Lily)................... 1.50 Hibiscus, Härdy Crimson Eye.......... 1.25 Hibiscus, Militaris ............. 1.25 Hibiscus, Moschentos............. 1.25 Hollyhock, Double............... 1.00 Hollyhock, Allegheny.............. 1.00
10.00 8.00 8.00 8.00

15.00

8.00

10.00

10.00

8.00

8.00

8.00

8.00

8.00

8.00

10.00

8.00

8.00

5.00

8.00

10.00

8.00

8.00

8.00

12.00

18.00

12.00

10.00

10.00

10.00

10.00

10.00

8.00

.8 .00

8.00

7.00

7.00 
HARDY PLANTS, BULBS AND ROOTS-Continued Per Per

Doz. 100

Iberis Sempervirens' Hardy Candytuft. . . $1.50 \quad 10.00$

Iris Kaempferi, named . . . . . . . . . 1.50 12.00

Iris Kaempferi mixed, extra heavy...... $1.50 \quad 12.00$

Iris Germanica, mixed.............. $.75 \quad 5.00$

Iris Germanica, named............ . .85 $\quad 6.00$

Gaillardia Grandiflora .............. $1.50 \quad 10.00$

Genista tinctoria, bright yellow pea-shaped

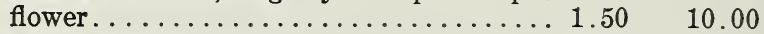

Lavender (pot plants)............ $1.00 \quad 7.00$

Lamium purpureum ............. $1.25 \quad 8.00$

Lathyrus Latifolius, Perennial Peas, pink

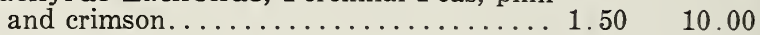

Lupinus polyphyllus............. $1.25 \quad 8.00$

Lychnis, Chalcedonica and Viscaria Splendens fl. pl. .................... 1.50

Lythrum roseum superbum.......... 1.25

Monarda didyma............... 1.25

Myosotis (Forget-me-not) alpestris and

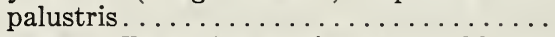

Pansies. Extra fine strains, strong bloom-

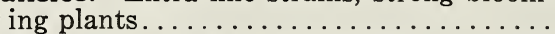

10.00

8.00

8.00

5.00

$.50 \quad 3.00$

Papaver orientale (Oriental Poppy), brilliant scarlet.................. 1.50

Iceland Poppy Orange, Yellow and White 1.25

Pardanthus Sinensis. Blackberry Lily. . 1.50

Pentstemon Coerulea.............. 1.50

Perennial Pea, Lathyrus, pink and crimson 1.50

Peonies, fine collection........... 5.00

Peonies, White................. 3.75

Perennial Phlox, 2-inch pots plants ready

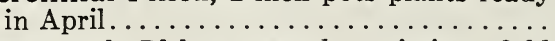

Perennial Phlox named varieties, field grown, white pink and purple............ 1.50

Phlox Subulata, white, pink eye...... 1.50

Pinks, assorted varieties, 21/2-inch pots.... .60

Pinks, assorted varieties clumps ........ 1.50

Platycodon grandiflorum, blue and white. 1.50

Physostegia Virginica, white and bright pink.................... 1.50

Pyrethrum, mixed colors........... 1.50

Rudbeckia (Cone Flower), Golden Glow . . 1.50

Rudbeckia Newmani and purpurea...... 1.50

Sage, Broad-leaved, pot plants ......... .85

Salvia pratensis, flower spikes of deep blue. 1. 50

Sedum in variety............... 1.50

Shasta Daisy, strong field grown . . . . . 1.25

Stokesia cyanea................. 1.50

Sweet William, assorted varieties....... 1.00

Thermopsis Caroliniana .............. 1.50

Veronica spicata, Rupestris, bright blue

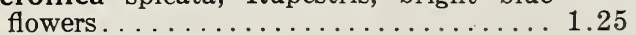

Violet, Snowflake............... .75

\section{VICK'S MOSS ROSES}

Henry Martin. Deep rosy carmine.

Madam de la Rochlambert. Bright pink.

Princess Adelaide. Pale rose, of good form.

White Bath. Best of the white Moss Roses. 


\section{VICK'S HARDY ROSES}

\section{Hardy Perpetuals}

Each, 50c.; doz., $\$ 5.00$; per $100, \$ 35.00$

Anna De Diesbach. Lovely shell pink.

Frau Karl Druschki (White American Beauty). Snow white flowers of immense size and beautiful form; one of the best bloomers of the Hardy Perpetual division. Very strong, vigorous growers.

General Jacqueminot. Brilliant red, large and full, very fragrant. Hardy and robust.

Mrs. John Laing. Shell pink. Easily one of the best pinks in the Hardy Perpetual class. Flowers are beautifully formed, finely colored, very fragrant. Long stemmed.

Paul Neyron. One of the largest of all rose blooms. Clear beautiful pink. Blooms all summer. Stems almost thornless.

Soliel d'Or or Sun of Gold. Golden yellow, shaded red. Flowers large and full. Free blooming and extremely hardy.

Ulrich Brunner. Brilliant cherry red, large flowers.

\section{EVERBLOOMING HYBRID TEA ROSES}

Each, 50c.; doz., $\$ 5.00$; per $100, \$ 35.00$

Columbia. Peach blow pink, deepening to rose pink.

Dorothy Page Roberts. Coppery Pink, suffused apricot yellow.

Gruss an Teplitz. A hardy free-growing rose with sweetly fragrant, dazzling fiery crimson blooms on long stems.

Francis Scott Key. Deep red; flowers large and well formed.

Hoosier Beauty. Crimson scarlet, darker shadings, velvety texture.

Irish Fireflame. Old gold, flushed crimson. Buds long and slender.

Ophelia. Salmon flesh shaded rose with chamois center. One of the best Hybrid Teas.

White Killarney. Pure white from the pink parent.

Lady Hillingdon. Growth is strong and vigorous, new foliage and stems dark violet, changing to deep green. Flower beautiful apricot yellow.

Los Angeles. One of the most beautiful and popular new American roses. Flame pink shaded coral and translucent gold.

Radiance. New brilliant carmine pink with yellow shadings at base of petals.

Red Radiance. Like Radiance except that it is a deep red color.

Killarney Pink. Famous pink, especially popular for its long pointed buds.

VICK'S HARDY CLIMBING ROSES

Doz. Per

100

Dorothy Perkins. Flowers very double, pink ........................ $\$ 2.75 \$ 20.00$

Red Dorothy Perkins. Decided improvement over Crimson Rambler.......... 2.75 20.00

Lady Gay. Delicate cherry pink, fragrant. $2.75 \quad 20.00$

Tausendschon. Strong plants. Delicate pink, large flowers in clusters.......... $3.50 \quad 25.00$ Write for prices on any varieties wanted not listed here. 


\section{Rambler Roses}

Two years old, doz., $\$ 2.75$; per $100, \$ 20.00$

Crimson Rambler. Old favorite bright crimson.

White Rambler. Great clusters of white flowers.

Pink Rambler. Large clusters of pink flowers.

Yellow Rambler. Very prolific, yellow flowers.

\section{HARDY VINES AND SHRUBS}

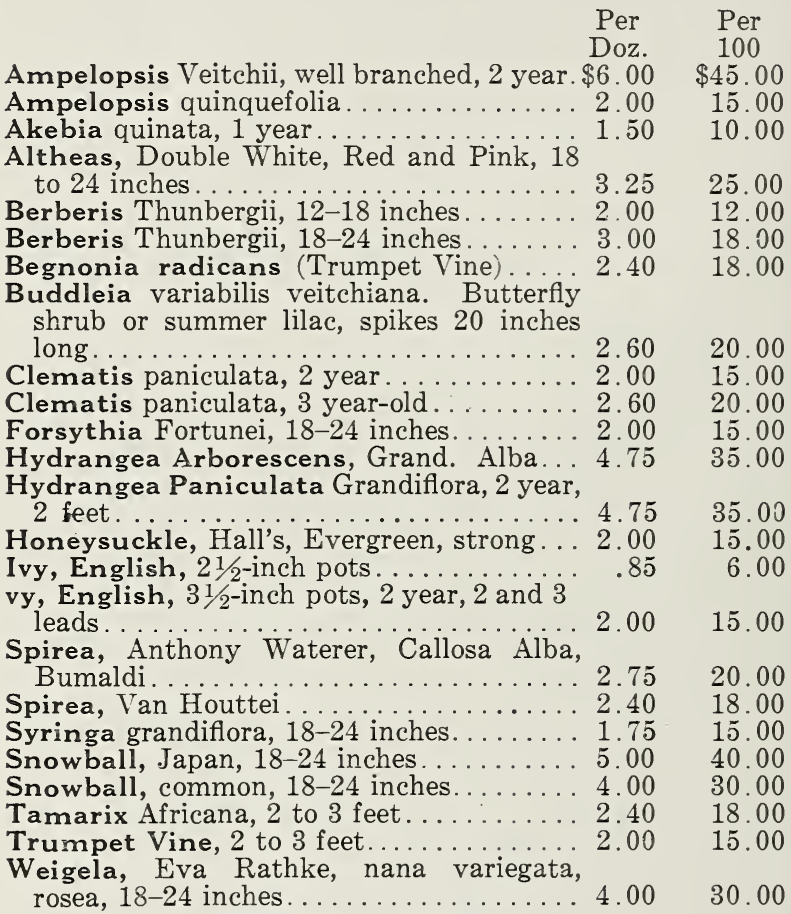

\section{TENDER BULBS AND TUBERS}

Per Per

Doz. $\quad 100$

Amaryllis Johnsoni, first size each, 25c. \$2.60 \$20.00 Childanthus fragrans.............. $1.25 \quad 8.00$

Caladium esculentum, $5-7$ inches..... $.60 \quad 4.50$

Caladium esculentum, medium size, 7-9 inches....................... 85

Caladium esculentum, extra large size, $9-$ 11 inches................... $1.75 \quad 12.00$

Caladium esculentum, mammoth size, 11 -12 inches. . . . . . . . . . . . . . . 2.00

Calla, Spotted (Richardia albo-maculata) . . 2.60

Cannas. Dormant roots. Alemania, Alphonse Bouvier, Burbank, Charles Henderson, Florence Vaughan, Mademoiselle Berat, President Faivre, Robusta per

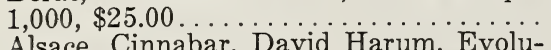
Alsace, Cinnabar, David Harum, Evolu-
tion, Fairfield, Italia, J. D. Cabos, Jean Tissot, Mme. Crozy, Souvenir de Antoine Crozy per $1,000, \$ 30.00 \ldots \ldots \ldots \ldots \ldots$. 
TENDER BULBS AND TUBERS-Continued.

Per Per

Doz. $\quad 100$

Giant Ismene, a beautiful white Amaryllis $\$ 1.50$

$\$ 12.00$

Hyacinthus candicans, first size per 1,000 , $\$ 20.00 \ldots \ldots \ldots \ldots \ldots \ldots \ldots \ldots \ldots .50 \ldots \ldots \ldots$

Madeira Vine, extra large selected tubers.. $\quad .75 \quad 5.50$

Oxalis, Deppii and lasiandra per 1,000, $\$ 7.50 \ldots \ldots \ldots \ldots \ldots \ldots \ldots$

Tuberose, Double Pearl, 4 to 6 inches, per $1,000, \$ 30.00 \ldots \ldots \ldots \ldots \ldots \ldots \ldots \ldots$

\section{VICK'S TENDER PLANTS}

Abutilons, Eclipse and Golden Fleece... \$ .85

Achania Malvaviscus.............. $\quad .75$

$\$ 6.00$

5.00

Alternantheras, yellow and red, per 1,000, $\$ 35.00 \ldots \ldots \ldots \ldots \ldots \ldots \ldots \ldots .6 \ldots$

Asparagus Sprengeri, 3 inch pots . . . . 1.25

Asparagus Plumosus nanus, 3 inch pots. 1.25

Begonias (flowering sorts). Chatelaine

and Semperflorens........................... Erdody, Rubrum, President Carnot and other leading varieties. $21 / 2$-inch pots. . . 1.50

Calceolaria, Creole Queen............. .85

Calla, Little Gem . . . . . . . . . . . . . . . . 85

Cannas. Pot plants are ready about April 1st. For list of varieties see "Tender

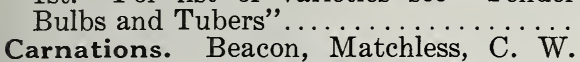
Ward, White Enchantress, Enchantress Supreme, Doris, Pink Matchless, Red Matchless.

Carex Japonica, variegata (Japanese

Grass) ........................ . .85

Chrysanthemums. In variety . . . . . . . Hardy Pompons, all best varieties. . . . . $\quad .75$

Coleus. Crimson Velvet, Duneira, Snowcloud, Firebrand, Sensation and other leading varieties...................

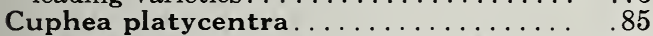

Cyperus alternifolius, $21 / 2$-inch pots.... .85

Cyperus alternifolius, 3 -inch pots. . . . 1.50

Dracaena indivisa, 2 -inch pots . . . . . . .60

Dracaena indivisa, 4 -inch pots . . . . . 2.75

Dracaena indivisa, 5 -inch pots . . . . . . 4.75

Ferns. Boston, 21/2-inch pots, strong . . . 1.25 Teddy, Jr. $21 / 2$-inch pots, strong . . . . . . 1.25

Assorted, for fern dishes, from $21 / 2^{-i n c h}$

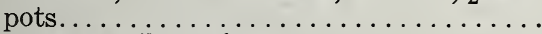

Fuchsias. In variety . . . . . . . . .

Feverfew, Little Gem, White..........

Geraniums, in variety, S. A. Nutt, Beaute Poitevine, Alphonse Ricard, Madame de Countesse de Baum, Mrs. Richard F. Gloede and other leading varieties, $2 \frac{1}{2}$ -

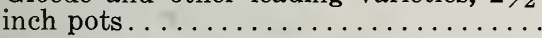

Geraniums, 3-inch pots . . . . . . . . 1.25

Geraniums, $31 / 2$-inch pots . . . . . . . $1.75 \quad 12.00$

The above is just a partial list of our Hardy and Tender Plants, Bulbs, Roots, Roses, „etc. If interested in any items not found here, write us. 


\section{HORTICULTURAL REQUISITES}

\section{Glazola (Glazing Cement)}

A perfect compound for glazing, reglazing and repair work on greenhouses and sash. It is superior to putty, as it never gets hard or brittle. It will outlast putty three or four times-no loose or sliding glass to bother for many years. It contracts and expands with the greenhouses, thereby eliminating broken glass to a minimum. Its elasticity remains under atmospheric conditions regardless of how hot or cold the weather may be. Glazola is always ready for instant use and will not harden on your hands, and we sell it under a money-back guarantee. Its color is black, being made from long fibre asbestos and elastic gums. In glazing, three gallons will set one thousand feet of glass. We offer you this Glazola Cement at the following prices: Thirtyfive and 55-gal. steel drums, $\$ 1.50$ per gal.; 5-gal. cans, $\$ 1.70$ per gal.; gal. can, $\$ 1.75 ; 1$ Glazing Gun gratis with each 35 or 55-gal. drum. Extra one-hand Glazing Guns, $\$ 5.00$ each.

Labels (Wooden)

Painted Pot Labels

4 inch per $1,000 \ldots \$ 1.75$

$41 / 2$ " " " " . . . 2.00

5 “ “ “. $\ldots 2.20$

6 " " " 6 . 62.60

Painted pot labels, packed 1,000 to package, 8 and 10 -inch garden labels, 250 to package, 12 -inch 100 to package.

\section{Simplex Weatherproof Plant Labels}

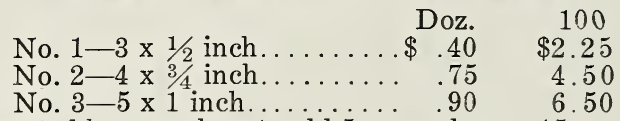

If wanted by parcel post, add 5 c. per doz. or $15 \mathrm{c}$. per $10 \mathrm{~J}$. Tree Labels

Four inch, painted and copper wired, 100, 40c.; 1,000, $\$ 2.60$.

\section{Grafting Wax}

One-fourth lb., 12c.; 1/2 lb., 18c.; 1 lb., 30c.

\section{Raffia}

The demand for Raffia is speedily growing, largely indused by the attention given to basket making in public and industrial schools. Dealers who do not carry a full line of Raffia are neglecting opportunities to increase sales materially. Samples of Raffia on application. Colors, red, pink, emerald green, yellow, black, dark navy, golden brown, dark brown, Christmas red, light orange and white. Prices: $1 / 4$ lb., 35c.; $1 / 2$ lb., 75c.; 1 lb., $\$ 1.10$. Natural, per lb., 20c.; 5 lbs. or over, 19c. per lb.

PAPER FLOWER POTS (Neponset Waterproof)

For starting plants in hot beds or greenhouses that are to be transplanted to the field.

Not less than 500 at the 1,000 rate. Prices F. O. B. Rochester, N. Y.

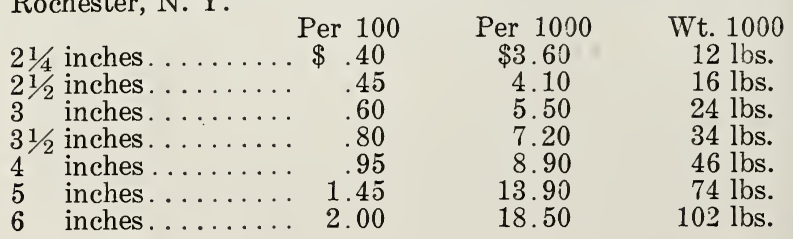




\section{PULVERIZED SHEEP MANURE}

We believe we have the best brand of Pulverized Sheep Manure obtainable. It is strictly a pure animal product with the addition of no commercial fertilizer to bring it up to high chemical analysis. Our sheep manure is co nposed of cleanings from wool and contains a minimum amount of dirt and foreign matter which is so common in the ordinary stock yards products. It also has an oily quality not found in the ordinary sheep manure. This makes it much more pleasant to use, making it less dry and powdery.

Sheep Manure is used by all florists and growers both for indoor and outdoor use. We use it in our own greenhouses in large quantities for mixing with potting soil. For outdoor use it is very convenient, comparatively odorless and produces quicker and better results than stable manure without the unsightly appearance of the latter. Per $100 \mathrm{lbs}$., $\$ 2.50$; 500 lbs., $\$ 12.00 ; 1,000$ lbs., $\$ 22.00 ; 1$ ton, $\$ 42.00$.

\section{SPRAYERS}

AUTO-SPRAY No. 1

The Auto-Spray No. 1 is strongly built and guaranteed to be satisfactory and without defect. Will handle all solutions, including whitewash, and can be used in all kinds of work. All parts are instantly accessible and easily replaced. Equipped with 2 feet of $1 / 2$-inch hose, nozzle for fine spray, cap for solid stream and either stop-cock or our patented self-cleaning shut-off "auto-pop" is orlered.

Auto-Spray No. 1-3

Brass tank with auto-pop.......\$7.13

Auto Spray No. 1-D

Galvanized tank with auto-pop..... 4.83

Auto Spray Nozzle.................. .45

Extension Pipe, solid brass, 2 feet. $\quad .40$

Elbow Extension, solid brass . ..... . .23

Brass Strainer. .................. .90

AUTO-SPRAY No. 26

A continuous atomizer of unusual merit. The pressure generated on the down stroke of the plunger allows this sprayer to produce a continuous mist-like spray. This is a decided advantage over ordinary intermittent atomizers as spraying can be done more rapidly and with less exertion. The container can be detached for refilling and cleaned by simply unscrewing from the pump cylinder. By removing the container the siphon tube is easily and quickly cleaned. Two nozzles are supplied, one for straight and one for angular spraying.

Auto Spray No. 26-A

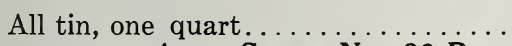
$\$ 7.50$

Per Doz.

Auto Spray No. 26-B

Tin pump. Brass tank, one quart... 9.50

Auto Spray No. 26-C

All brass, one quart............ 11.31

\section{MODEL “B” DUSTER}

A simple, inexpensive and efficient duster for applying all kinds of insecticides in powdered form. The use of powdered insecticides is becoming more and more common and proves very effective. This duster will distribute material evenly and finely as fast as a man can readily walk down a row. Each, 90c.; doz., $\$ 9.25$. 


\section{INSECTICIDES}

Directions for using printed on each package.

\section{Arsenate of Lead Paste}

The standard poison for all leaf-eating insects. One lb., 35c.; 5 lbs., \$1.50; 25 lbs., \$5.25; 50 lbs., \$9.00; 100 lbs., $\$ 17.50$.

\section{Arsenate of Lead, Powdered}

One to one-and-a-half pounds for 50 gallons water. One lb., 45c.; 5 lbs., $\$ 2.00$; 10 lbs., $\$ 3.75 ; 25$ lbs., $\$ 8.00 ; 50$ lbs., $\$ 14.00 ; 100$ lbs., $\$ 26.00$.

\section{Bordo-Lead (Paste)}

A combined insecticide and fungicide. One lb., 35c.; 5 lbs., \$1.35; 10 lbs., $\$ 2.25$; 25 lbs., $\$ 4.75 ; 50$ lbs., $\$ 9.00 ; 100$ lbs., $\$ 17.00$.

\section{Bordeaux Mixture Powder}

Recommended by all the leading experts for general spraying, principally for mildew, blight and other fungus diseases. One lb., 30c.; 5 lbs., $\$ 1.00 ; 25$ lbs., $\$ 3.75$.

\section{Black Leaf -40}

A concentrated solution of nicotine-sulphate which does not evaporate, like "free" nicotine, in ordinary temperatures. One-ounce bottle, 25 c.; $1 / 2$-lb. can, $\$ 1.10 ; 2-1 b$. can, $\$ 3.25$; $10-\mathrm{lb}$. can, $\$ 13.50$.

\section{Carbola}

A white paint and powerful disinfectant in powder form. Quickly mixed with water. Apply with brush or spray. Five lbs., 60c.; 10 lbs., $\$ 1.00$; 50 lbs., $\$ 4.50$.

\section{Cyanogas}

A new material for generating hydrocyanic gas. The gas is evolved merely by exposure to the atmosphere, making it extremely easy to use. It is by far the most economical method of fumigating greerhouses yet devised, and when directions are followed it is a most effective means of ridding plants of white fly, aphis and many other insects.

Cyanogas G fumigant is recommended for greenhouse fumigating. Cyanogas A dust is more finely pulverized, gives off its gas more rapidly and may be used in a duster for use against rats, moles, ants, woodchucks, ground hogs, squirrels and for orchard dusting when diluted.

Both grades, 1 lb., 60 c.; 5 lbs., $\$ 2.50 ; 25$ lbs., $\$ 8.50 ; 100$ lb. drums, $\$ 25.00$.

\section{Hellebore}

Pure and fresh; used largely for currant worms, etc. One-fourth lb., 18c.; $1 / 2$ lb., 30c.; 1 lb., 50c.

\section{Lime Sulphur, Powdered}

Most economical form for shipping. Just as good as liquid. One-and-one-half pounds will make 5 gallons of solution of standard strength. One lb., 35c.; 5 lbs., $\$ 1.30$; 10 lbs., $\$ 2.10$.

\section{Melrosine}

An effective remedy for rose bugs. Used in any sprayer diluted with 20 to 25 gallons of water. Pint, $\$ 1.00$; $1 / 2$ gal., $\$ 3.25$; gal., $\$ 6.00$.

\section{Nicofume, Liquid}

Used for same purposes as Black Leaf-40. Guaranteed 40 per cent Nicotine. One-fourth lb., 75c.; 1 lb., $\$ 2.00 ; 4$ lbs., $\$ 7.50 ; 8$ lbs., $\$ 14.50$.

\section{Nicotine, Hall's Free}

For effective greenhouse spraying and vaporizing. Onehalf lb., $\$ 1.40 ; 2$ lbs., $\$ 4.25 ; 8$-lb. can, $\$ 15.00$. 


\section{INSEGITIDES (Con't)}

\section{Nicotine Fumigator (Hall's)}

Specially prepared compound saturated with pure nicotine, burns easily and evenly. One lb., $\$ 1.25 ; 21 / 2 \mathrm{lbs}$, $\$ 2.50 ; 10$ lbs., $\$ 8.50$.

\section{Nicotine Sulphate (Hall's)}

Forty per cent nicotine guaranteed. One oz., 35c.; 1/2-lb. tins, $\$ 1.25 ; 2-\mathrm{lb}$. tins, $\$ 3.50$; 10 -lb. tins, $\$ 13.50$.

\section{Nicofume Paper}

Handy method of fumigating, packed in special friction top cans. Per can (24 sheets) $\$ 1.25 ; 1$-gross cans (144 sheets) $\$ 5.50$; 2-gross cans (288 sheets) $\$ 10.00$.

\section{Pyrox (Bordeau-Arsenate)}

A combined insecticide and fungicide for general use on apples, grapes and garden truck. Per 1 lb., 45c.; 5 lbs., $\$ 1.85 ; 10$ lbs., $\$ 3.25 ; 25$ lbs., $\$ 6.75 ; 50$ lbs., $\$ 12.00$.

\section{Paris Green}

A poisonous, unsoluble powder, used extensively for potato bugs, etc. Applied as a powder, mix with plaster or flour; in a solution, mix one pound in 200 to 300 gallons of water. One-fourth lb., 20c.; $1 / 2$ lb., 30c.; 1 lb., 55 c.

\section{Scalecide}

Used for the destruction of the San Jose Scale. Dilute at the rate of one gallon Scalecide to 20 gallons of water. Scalecide does not clog hose, nozzle or pump, and is pleasant to use. Fifty-five cents per quart; $\$ 1.40$ per gallon; $\$ 5.75$ per 5 gallons.

\section{Slug Shot}

Non-poisonous powder for potato bugs, cabbage lice and worms, currant worms, etc. One lb., 20c.; 5 lbs., 55c.; 10 lbs., $\$ 1.10$.

\section{Sulpho-Tobacco Soap}

Used as a wash for roses, trees and house plants. Also for insects on animals. Small size, 3-oz. cake, 12c.; doz., 90c.; large size, 8-oz. cake, 20c.; doz., \$2.25.

\section{Weed Killer (Liquid)}

Destroys weeds in paths, roads and gutters. Kills roots well as tops of all weeds and grass: One qt., $60 \mathrm{c}$; 1 gal., $\$ 1.45$; 5 gals., $\$ 6.00$.

\section{PEAT MOSS}

\section{For Florists, Nurserymen and Gardeners}

This is decomposed vegetable matter thoroughly dried, odorless, free from weeds and disease and granulated to convenient size. This material is well known in Europe, having been used for many years by successful growers, and has already found great favor in this country by the best growers, especially as more and more attention is being paid to the fact that productive soil requires large amounts of humus to replace that which is taken off in crops. Granulated Peat Moss furnishes humus in its purest form without the addition of disease spores and fungus which is found in all manure. It absorbs and retains ten times its weight of water, and the mixing of this material with potting soil and the addition of a little complete fertilizer will produce as good results as the very richest manure without the dangers from bacteria and disease. For mulching around evergreens, rhododendrons, shrubbery of all kinds, it is unexcelled. Packed in large bales about 21 x 40 inches, covering about 240 square feet, one inch deep, $\$ 3.00$ each; small bales for trial purposes, $\$ 1.00$ each. 
